\title{
Stability Theory of Difference Approximations for Mixed Initial Boundary Value Problems. II
}

\author{
By Bertil Gustafsson, Heinz-Otto Kreiss and Arne Sundström
}

\begin{abstract}
A stability theory is developed for general difference approximations to mixed initial boundary value problems.

The results are applied to certain commonly used difference approximations which are stable for the Cauchy problem, and different ways of defining boundary conditions are analyzed.
\end{abstract}

1. Introduction. Consider a first order system of partial differential equations

$$
\partial u(x, t) / \partial t=A \partial u(x, t) / \partial x+B u(x, t)+F(x, t)
$$

in the quarter-plane $0 \leqq x<\infty, t>0$. Here, $A$ and $B$ are constant square matrices and

$$
u(x, t)=\left(u^{(1)}(x, t), \cdots, u^{(n)}(x, t)\right)^{\prime}, \quad, F(x, t)=\left(F^{(1)}(x, t), \cdots, F^{(n)}(x, t)\right)^{\prime}
$$

are vector functions. Furthermore, $A$ is Hermitian and of the form:

$$
A=\left(\begin{array}{cc}
A_{1} & 0 \\
0 & A_{2}
\end{array}\right) \text { with } A_{1}<0, A_{2}>0
$$

The solution of (1.1) is uniquely determined if we prescribe initial values for $t=0$ :

$$
u(x, 0)=f(x), \quad 0 \leqq x<\infty,
$$

and boundary conditions for $x=0$ :

$$
u^{\mathrm{I}}(0, t)=S u^{\mathrm{II}}(0, t)+g(t), \quad t \geqq 0,
$$

where

$$
u^{\mathrm{I}}=\left(u^{(1)}, \cdots, u^{(l)}\right)^{\prime} \text { and } u^{\mathrm{II}}=\left(u^{(l+1)}, \cdots u^{(n)}\right)^{\prime}
$$

correspond to the partition of $A$, and $S$ is a rectangular matrix.

We want to solve the above problem by difference approximation. For that reason, we introduce a time-step $k>0$, a mesh width $h=1 / N$ where $N$ is a natural number, and divide the $x$-axis into intervals of length $h$. As usual, we assume that $k / h=\lambda=$ const. Let $p, q, r$, and $s$ be natural numbers and use the notation

$$
v_{\nu}(t)=v\left(x_{\nu}, t\right), \quad x_{\nu}=\nu h, \quad \nu=-r+1,-r+2, \cdots, 0,1, \cdots .
$$

We approximate (1.1) for $\nu=1,2, \cdots$ and $t=t_{\tau}=\tau k, \tau=s, s+1, \cdots$ by a

Received August 17, 1971.

AMS 1970 subject classifications. Primary 65M10.

Copyright @ 1972, American Mathematical Society 
consistent multistep method

$$
Q_{-1} v_{\nu}(t+k)=\sum_{\sigma=0}^{s} Q_{\sigma} v_{\nu}(t-\sigma k)+k F_{\nu}(t)
$$

Here,

$$
Q_{\sigma}=\sum_{j=-r}^{p} A_{j \sigma}(h) E^{j}, \quad E v_{\nu}=v_{\nu+1},
$$

are difference operators with matrix coefficients which depend smoothly on $h$. For the solution of (1.5) to be uniquely determined, it is necessary to specify initial values

$$
v_{\nu}(\sigma k)=f_{\nu}(\sigma k), \quad \sigma=0,1,2, \cdots, s ; \nu=-r+1,-r+2, \cdots,
$$

and, for $t=t_{\tau} \geqq s k$, boundary conditions

$$
v_{\mu}(t+k)=\sum_{\sigma=-1}^{s} S_{\sigma}^{(\mu)} v_{1}(t-\sigma k)+g_{\mu}(t), \quad \mu=-r+1, \cdots, 0,
$$

where

$$
S_{\sigma}^{(\mu)}=\sum_{i=0}^{q} C_{j \sigma}^{(\mu)} E^{i}
$$

are onesided difference operators, i.e. (1.7) expresses the solution at the boundary point $x_{\mu} \leqq 0$ in terms of the solution at interior points.

The aim of this paper is to generalize the stability theory of [1] to the nondissipative case. Furthermore, we shall also treat the case with two boundaries, i.e. consider the differential equations (1.1) for $0 \leqq x \leqq 1, t \geqq 0$. Then, we also have to specify boundary conditions for $x=1$. In this paper we will treat only the case with constant coefficients. However, the generalization to equations with variable coefficients poses no new difficulties because stability is always proved by the energy method.

We shall use the same notations as in [1] and assume that the reader is familiar with that paper.

2. Estimates for the Differential Equations. As a preliminary, we shall derive some estimates for the solutions of the differential equations. The reason why we derive these inequalities is that the same type of technique is later used to give estimates for the solutions of the difference approximations.

Let us introduce the $L_{2}$-scalar products

$$
\begin{aligned}
(u, v)_{x} & =\int_{0}^{\infty} u^{*}(x, t) v(x, t) d x, \quad(u, v)_{t}=\int_{0}^{\infty} u^{*}(x, t) v(x, t) d t, \\
(u, v)_{x, t} & =\int_{0}^{\infty} \int_{0}^{\infty} u^{*}(x, t) v(x, t) d x d t,
\end{aligned}
$$

and define the corresponding $L_{2}$-norms in the usual way by $\|u\|^{2}=(u, u)$. We denote the corresponding $L_{2}$-spaces by $L_{2}(x), L_{2}(t)$ and $L_{2}(x, t)$, respectively.

The following estimate is well known:

THEOREM 2.1. Let $F=g=0$. For every $f \in L_{2}(x)$, the problem (1.1)-(1.4) has a 
unique solution which belongs to $L_{2}(x)$ for every fixed $t$. Furthermore, there are real constants $\alpha_{0}, K_{0}$ such that for all $f$ and all $\alpha>\alpha_{0}$ an estimate

$$
\left\|e^{-\alpha t} u(x, t)\right\|_{x} \leqq K_{0} \downarrow \mid f(x) \|_{x}
$$

holds.

We can also prove

THEOREM 2.2. Let $f=F=0$. There is a constant $\alpha_{0} \geqq 0$ such that (1.1)-(1.4) has, for every $g(t) \in L_{2}(t)$, a unique solution $u(x, t)$ with $e^{-\alpha t} u(x, t) \in L_{2}(x, t)$ for $\alpha>\alpha_{0}$. Furthermore, there is a constant $K_{0}$ such that, for all $g$,

$$
\left\|e^{-\alpha t} u(0, t)\right\|_{t} \leqq K_{0}\left\|e^{-\alpha t} g(t)\right\|_{t},
$$

and

$$
\left(\alpha-\alpha_{0}\right)\left\|e^{-\alpha t} u(x, t)\right\|_{x, t}^{2} \leqq K_{0}^{2}\left\|e^{-\alpha t} g(t)\right\|_{t}^{2} .
$$

Proof. Here, we shall only prove the estimates. Let $u(x, t)$ be a solution with $e^{-\alpha t} u(x, t) \in L_{2}(x, t)$ for all sufficiently large $\alpha$. Introduce into (1.1)-(1.4) a new variable $w=e^{-\alpha t} u$. Then,

$$
\begin{aligned}
\partial w / \partial t & =A \partial w / \partial x+B w-\alpha w \\
w(x, 0) & =0, \quad w^{\mathrm{I}}(0, t)=S w^{\mathrm{II}}(0, t)+\tilde{g}(t),
\end{aligned}
$$

with $\tilde{g}=e^{-\alpha t} g$.

Defining now $w \equiv 0$ (and $\tilde{g}(t) \equiv 0$ ) for $t \leqq 0$, we can remove the initial line $t=0$ and consider (2.4) as a boundary value problem for $-\infty<t<\infty$. Fourier transforming (2.4) with respect to $t$ then gives us the system of ordinary differential equations

$$
s \hat{w}=A d \hat{w} / d x+B \hat{w}, \quad s=i \xi+\alpha, \hat{w}=\hat{w}(x, s),
$$

with boundary conditions

$$
\hat{w}^{\mathrm{I}}(0, s)=S \hat{w}^{\mathrm{II}}(0, s)+\hat{\tilde{g}} .
$$

Here,

$$
\hat{w}=\hat{w}(x, s)=(2 \pi)^{-1 / 2} \int_{-\infty}^{+\infty} e^{-i \xi t} w(x, t) d t=(2 \pi)^{-1 / 2} \int_{-\infty}^{+\infty} e^{-s t} u(x, t) d t .
$$

We can write (2.5) in the form

$$
d \hat{w} / d x=M \hat{w}, \quad M=s A^{-1}\left(I-s^{-1} B\right) .
$$

From (1.2), it follows that there is an $\alpha_{0}>0$ and a nonsingular transformation

$$
T=I+s^{-1} T_{1}+s^{-2} T_{2}+\cdots
$$

such that, for $\operatorname{Re} s>\alpha_{0}$,

$$
T M T^{-1}=\left(\begin{array}{cc}
M_{1} & 0 \\
0 & M_{2}
\end{array}\right) \text { with } M_{1}+M_{1}^{*}<0, M_{2}+M_{2}^{*}>0 .
$$

Introducing the transformed variable $\hat{y}=T \hat{w}$ into $(2.5 \mathrm{a})$, we obtain 


$$
d \hat{y} / d x=\left(\begin{array}{cc}
M_{1} & 0 \\
0 & M_{2}
\end{array}\right) \hat{y},
$$

with the solution

$$
\hat{y}^{\mathrm{I}}(x, s)=e^{M_{1} x} \hat{y}^{\mathrm{I}}(0, s), \quad \hat{y}^{\mathrm{II}}(x, s)=e^{M_{2} x} \hat{y}^{\mathrm{II}}(0, s) .
$$

The boundary conditions (2.6) get the form

$$
\left(T^{-1} \hat{y}\right)^{\mathrm{I}}=S\left(T^{-1} \hat{y}\right)^{\mathrm{II}}+\hat{g}
$$

which can also be written as

$$
\hat{y}^{\mathrm{I}}(0, s)=S \hat{y}^{\mathrm{II}}(0, s)+O\left(s^{-1}\right) \hat{y}(0, s)+\hat{\boldsymbol{g}} .
$$

In addition, we have by assumption

$$
\int_{-\infty}^{+\infty}\|\hat{y}(x, s)\|_{x}^{2} d \xi=\int_{-\infty}^{+\infty}\|T \hat{w}\|_{x}^{2} d \xi \leqq \text { const }\|w\|_{x, t}^{2}=\text { const }\left\|e^{-\alpha t} u(x, t)\right\|_{x, t}^{2}<\infty
$$

for all sufficiently large $\alpha$. Because $e^{M_{2} x}$ is exponentially increasing, we get $\hat{y}^{\mathrm{II}}(0, s)=0$ and (2.8) thus implies, for sufficiently large $\alpha_{0}$,

$$
|\hat{w}(0, s)|=\left|T^{-1} \hat{y}(0, s)\right| \leqq \text { const }|\hat{g} \hat{\mid}|
$$

Therefore, (2.2) follows from Parseval's relation.

Assume now that also $|B| \leqq \alpha_{0}$ and multiply (2.5) by $\hat{w}^{*}$. Then

$$
\begin{aligned}
\alpha\|\hat{w}\|_{x}^{2} & =\operatorname{Re}(\hat{w}, A d \hat{w} / d x)_{x}+\operatorname{Re}(\hat{w}, B \hat{w})_{x} \\
& =-\frac{1}{2} \hat{w}^{*}(0, s) A \hat{w}(0, s)+\operatorname{Re}(\hat{w}, B \hat{w})_{x} \geqq \text { const }|\hat{g}|^{2}+\alpha_{0}\|\hat{w}\|_{x}^{2},
\end{aligned}
$$

i.e.,

$$
\left(\alpha-\alpha_{0}\right)\|\hat{w}\|_{x}^{2} \leqq \text { const }|\hat{g}|^{2},
$$

and (2.3) follows again from Parseval's relation.

Finally, we shall prove

THEOREM 2.3. Let $f=0$. There is a constant $\alpha_{0}$ such that (1.1)-(1.4) has, for every $F \in L_{2}(x, t)$ and $g(t) \in L_{2}(t)$, a unique solution $u(x, t)$ with $e^{-\alpha t} u(x, t) \in L_{2}(x, t)$, $e^{-\alpha t} u(0, t) \in L_{2}(t)$ for $\alpha>\alpha_{0}$. Furthermore, there is a constant $K_{0}$ such that, for all $F$ and $g$,

$$
\begin{aligned}
\left(\alpha-\alpha_{0}\right)\left\|e^{-\alpha t} u(0, t)\right\|_{t}^{2}+ & \left(\alpha-\alpha_{0}\right)^{2}\left\|e^{-\alpha t} u(x, t)\right\|_{x, t}^{2} \\
& \leqq K_{0}^{2}\left(\left(\alpha-\alpha_{0}\right)\left\|e^{-\alpha t} g(t)\right\|_{t}^{2}+\left\|e^{-\alpha t} F(x, t)\right\|_{x, t}^{2}\right) .
\end{aligned}
$$

Proof. Again, we shall only prove the estimate. Let $u(x, t)$ be a solution for which $e^{-\alpha t} u(x, t) \in L_{2}(x, t), e^{-\alpha t} u(0, t) \in L_{2}(t)$ for all sufficiently large $\alpha$. Then, we can again introduce a new variable

$$
w=e^{-\alpha t} u \quad \text { for } t \geqq 0, \quad w=0 \quad \text { for } t<0,
$$

and get, instead of (2.5), (2.6),

$$
\begin{aligned}
s \hat{w} & =A d \hat{w} / d x+B \hat{w}+\hat{F^{\hat{w}}}, \quad s=i \xi+\alpha, \\
\hat{w}^{\mathrm{I}}(0, s) & =S \hat{w}^{\mathrm{II}}(0, s)+\hat{g} .
\end{aligned}
$$


Here, $w(x, s) \in L_{2}(x)$ for sufficiently large $\alpha$. Now let

$$
H=\left(\begin{array}{cc}
\rho I & 0 \\
0 & I
\end{array}\right), \quad \rho=\text { const }>0,
$$

be a matrix. From (2.10), we get, by partial integration,

$$
\begin{aligned}
\alpha(\hat{w}, H \hat{w})_{x} & =\operatorname{Re}(\hat{w}, H A d \hat{w} / d x)_{x}+\operatorname{Re}(\hat{w}, H B \hat{w})_{x}+\operatorname{Re}(\hat{w}, H \tilde{F})_{x} \\
& \leqq R+|B|\|\hat{w}\|_{x}^{2}+\frac{1}{2} \alpha(\hat{w}, H \hat{w})_{x}+\frac{1}{2} \alpha^{-1}\|\tilde{F}\|_{x}^{2},
\end{aligned}
$$

where

$$
R=-\frac{1}{2}\left(\hat{w}^{\mathrm{I}}(0, s)\right)^{*} \rho A_{1} \hat{w}^{\mathrm{I}}(0, s)-\frac{1}{2}\left(\hat{w}^{\mathrm{II}}(0, s)\right)^{*} A_{2} \hat{w}^{\mathrm{II}}(0, s) .
$$

Using the boundary conditions, we can choose $\rho$ so small that

$$
R \leqq-\frac{1}{2} \delta|\hat{w}(0, s)|^{2}+\frac{1}{2} \delta^{-1}|\hat{g}|^{2} .
$$

Here, $\delta>0$ is a constant. Therefore, we get

$$
\alpha\left((\rho \alpha-2|B|)|| \hat{w} \|_{x}^{2}+\delta|\hat{w}(0, s)|^{2}\right) \leqq\left\|\hat{F^{\wedge}}\right\|_{x}^{2}+\delta^{-1} \alpha|\hat{g}|^{2},
$$

and (2.9) follows from Parseval's relation with $\alpha_{0}=2|B| / \rho$.

3. The Stability Definition. While, for the differential equations, all estimates (2.1)-(2.3), (2.9) hold at the same time, this is not true of the corresponding estimates for the difference approximations. As a consequence, there are several ways to define stability of difference approximations. We shall discuss some possible definitions. (Questions of convergence will be treated in a coming paper.) Let $l_{2}(x)$ denote the space of all gridfunctions $v_{\nu}=v\left(x_{\nu}\right), x_{\nu}=\nu h, \nu=-r+1,-r+2, \cdots, 0,1, \cdots$ with $\sum_{\nu=-r+1}^{\infty}\left|v_{\nu}\right|^{2} h<\infty$ and define the scalar product and norm by

$$
(u, v)_{x}=\sum_{\nu=-r+1}^{\infty} u_{\nu}^{*} v_{\nu} h, \quad\|u\|_{x}^{2}=(u, u)_{x} .
$$

We define $l_{2}(t)$ and $l_{2}(x, t)$ in the corresponding way and denote by

$$
\begin{aligned}
(u, v)_{t} & =\sum_{\tau=0}^{\infty} u^{*}\left(t_{\tau}\right) v\left(t_{\tau}\right) k, \quad\|u\|_{t}^{2}=(u, u)_{t}, \quad t_{\tau}=\tau k, \\
(u, v)_{x, t} & =\sum_{\tau=0}^{\infty} \sum_{\nu=-r+1}^{\infty} u_{\nu}^{*}\left(t_{\tau}\right) v_{\nu}\left(t_{\tau}\right) h k, \quad\|u\|_{x, t}^{2}=(u, u)_{x, t}
\end{aligned}
$$

the corresponding norms and scalar products.

Remark. We use the same notation as in the continuous case. There is no risk for confusion because from now on $(u, v)_{x},(u, v)_{t}$ and $(u, v)_{x, t}$ are always defined by (3.1)-(3.3), respectively.

Assumption 3.1. The Eqs. (1.5)-(1.7) can be solved boundedly for $v(t+k)$, i.e., there is a constant $K_{1}>0$ such that, for every $G \in l_{2}(x)$, there is a unique solution $w \in l_{2}(x)$ of

$$
\begin{aligned}
Q_{-1} w_{\nu} & =G_{v}, & \nu & =1,2, \cdots, \\
w_{\mu}-S_{-1}^{(\mu)} w_{1} & =g_{\mu}, & \mu & =-r+1, \cdots,-1,0,
\end{aligned}
$$


with

$$
\|w\|_{x}^{2} \leqq K_{1}\left(\|G\|_{x}^{2}+h \sum_{\mu=-r+1}^{0}\left|g_{\mu}\right|^{2}\right) .
$$

(Osher [5] has given conditions such that this assumption is fulfilled.) The first possibility is to use the same definition as for the Cauchy problem (see also Theorem 2.1).

Definition 3.1. Consider the difference approximation (1.5)-(1.7) with $g_{\mu}=F_{\nu}=0$, $\mu=-r+1, \cdots, 0, \nu=1,2, \cdots$. The approximation is stable if there are constants $K_{0}>0, \alpha_{0} \geqq 0$ such that, for all $t=t_{\tau}=\tau k$, all $\alpha>\alpha_{0}$, all $h$ and all $f$, an estimate

$$
\left\|e^{-\alpha t} v\right\|_{x}^{2} \leqq K_{0}^{2} \sum_{\sigma=0}^{s}\|f(\sigma k)\|_{x}^{2}
$$

holds.

In the same way as for the Cauchy problem, the analogue of Duhamel's principle gives us

LEMMA 3.1. If the difference approximation is stable in the sense of (3.4), then the estimate

$$
\left\|e^{-\alpha t} v(t)\right\|_{x}^{2} \leqq K_{0}^{2}\left(\sum_{\sigma=0}^{s}\|f(\sigma k)\|_{x}^{2}+\left(\alpha-\alpha_{0}\right)^{-1} \sum_{\tau=s}^{t / k}\left\|e^{-\alpha(\tau+1) k} F(\tau k)\right\|_{x}^{2} k\right)
$$

is valid for the inhomogeneous problem with $g_{\mu}=0, \mu=-r+1, \cdots, 0$.

The trouble with Definition 3.1 is that it is very difficult to develop a general stability theory. The following definition is more useful:

Definition 3.2. Assume that $f_{\nu}(\sigma k)=0, \nu=-r+1, \cdots, 0,1, \cdots ; \sigma=0$, $\cdots, s$. The approximation is stable if, instead of (3.4), an estimate

$$
\left(\frac{\alpha-\alpha_{0}}{\alpha k+1}\right)^{2}\left\|e^{-\alpha t} v\right\|_{x, t}^{2} \leqq K_{0}^{2}\left(\left\|e^{-\alpha(t+k)} F\right\|_{x, t}^{2}+h^{-1} \sum_{\mu=-r+1}^{0}\left\|e^{-\alpha(t+k)} g_{\mu}\right\|_{t}^{2}\right)
$$

holds. (We set $F_{\mu} \equiv 0$ for $\mu \leqq 0$ and $t<s k$, a convention we shall always use.) If the approximation is stable according to Definition 3.1, then it is also stable with respect to Definition 3.2. This follows from Lemma 3.1. We conjecture that the two definitions are equivalent.

A stability definition should preferably be such that one can use it "pointwise" for equations with variable coefficients and that one can check the stability as easily as possible. The last definition fulfills the first condition. However, necessary and sufficient stability conditions are not simple. To demonstrate this, we shall derive such conditions for dissipative approximations. In general, we use rather the stronger.

Definition 3.3. Assume that $f(\sigma k)=0, \sigma=1,2, \cdots, s$. The approximation is stable if, instead of (3.6),

$$
\begin{aligned}
\left(\frac{\alpha-\alpha_{0}}{\alpha k+1}\right) \sum_{\mu=r+1}^{0}\left\|e^{-\alpha t} v_{\mu}\right\|_{t}^{2}+\left(\frac{\alpha-\alpha_{0}}{\alpha k+1}\right)^{2}\left\|e^{-\alpha t} v\right\|_{x, t}^{2} \\
\\
\leqq K_{0}^{2}\left(\left(\frac{\alpha-\alpha_{0}}{\alpha k+1}\right) \sum_{\mu=-r+1}^{0}\left\|e^{-\alpha(t+k)} g_{\mu}\right\|_{t}^{2}+\left\|e^{-\alpha(t+k)} F\right\|_{x, t}^{2}\right)
\end{aligned}
$$

holds.

Definition 3.3 is obviously stronger than Definition 3.2 and is suggested by Theorem 2.3. In this case, necessary and sufficient stability conditions are as simple 
as possible. Most difference approximations in use are stable in this sense, although there are some which are only stable in the sense of Definition 3.2. In practice, it is not too important in which sense the approximation is stable, once an estimate of type (3.6) or (3.7) has been found.

The assumption $f(\sigma k)=0, \sigma=1, \cdots, s$, is unimportant. As an example, consider the case $g=F=0$ for which we get

THEOREM 3.1. Let $g=F=0$ and assume that the approximation is stable with respect to Definition 3.2 or 3.3. Then, there is a constant $K_{1}$ such that

$$
\left(\frac{\alpha-\alpha_{0}}{\alpha k+1}\right)^{2}\left\|e^{-\alpha t} v\right\|_{x, t}^{2} \leqq h^{-1} K_{1} \sum_{\sigma=0}^{s}\|f(\sigma k)\|_{x}^{2}
$$

and, therefore,

$$
\left(\frac{\alpha-\alpha_{0}}{\alpha k+1}\right)^{2}\left\|e^{-\alpha t} v\right\|_{x}^{2} \leqq(h k)^{-1} K_{1} \sum_{\sigma=0}^{8}\|f(\sigma k)\|_{x}^{2}
$$

Proof. Let $w$ be the function satisfying (1.7) and with

$$
w_{\nu}(t)=0 \quad \text { for } \nu \geqq 0, t \geqq s k, \quad w_{\nu}(t)=f_{\nu}(\sigma k) \quad \text { for } \nu \geqq-r+1, t=\sigma k .
$$

Then, $y=v-w$ is the solution of (1.5)-(1.7) with homogeneous initial values and boundary conditions and a function $F$ for which $\|F\|_{x, t}^{2} \leqq$ const $h^{-1} \sum_{\sigma=0}^{s}\|f(\sigma k)\|_{x}^{2}$. Therefore, (3.8) follows easily from (3.6) or (3.7).

In the same manner, it is easy to show that it is sufficient to study the case $g_{\mu}=0$, $\mu=-r+1, \cdots, 0$, in Definition 3.2.

THEOREM 3.2. If an estimate (3.6) holds for the case $g_{\mu} \equiv 0$, the approximation is stable according to Definition 3.2.

Proof. Let $w$ be the function defined by $w_{\mu}(t+k)=g_{\mu}(t)$ for $t \geqq s k, \mu=$ $-r+1, \cdots, 0 ; w_{v}(t)=0$ otherwise. Then, $y=v-w$ is the solution of (1.5)-(1.7) with homogeneous initial and boundary conditions, and (3.6) follows directly.

The generalization of the above definitions to problems with two boundaries is obvious.

In certain applications, it is interesting to know whether there are any exponentially growing solutions or not. We may then use

THEOREM 3.3. If $\alpha_{0}=0$ in (3.6) or (3.7), then there are no exponentially growing solutions.

Proof. Choose $\alpha=T^{-1}$ for every fixed time $T>0$.

4. The Resolvent Condition. In this section, we want to show how the question of stability can be reduced to an estimate of the solution to a resolvent equation. Connected with the Definitions 3.2, 3.3, there is the following resolvent equation:

$$
\left(Q_{-1}-\sum_{\sigma=0}^{8} z^{-\sigma-1} Q_{\sigma}\right) \hat{w}_{\nu}=\tilde{F_{\nu}}, \quad \nu=1,2, \cdots,
$$

with the boundary conditions

$$
\hat{w}_{\mu}-\sum_{\sigma=1}^{s} z^{-\sigma-1} S_{\sigma}^{(\mu)} \hat{w}_{1}=\tilde{g}_{\mu}^{\hat{\mu}}, \quad \mu=-r+1, \cdots, 0 .
$$

Here, $z$ is a complex number and $\hat{w} \in l_{2}(x)$. 
We start with the Ryabenkii-Godunov condition:

LEMMA 4.1. Let the approximation be stable in any sense. Then the homogeneous equations (4.1), (4.2) have for $|z|>e^{\alpha_{0} k}$ only the trivial solution $\hat{w} \equiv 0$.

Proof. Assume that $\hat{w} \in l_{2}(x)$ is a nontrivial solution. Then $v\left(x_{\nu}, t\right)=z^{t / k} \hat{w}_{\nu}$ is a solution of the difference approximation with $F=g=0$ and $f_{\nu}(\sigma k)=z^{\sigma} w_{\nu}$. This solution grows faster than $e^{\alpha_{0} t}$, which is impossible by Theorem 3.1.

We now have two theorems, relating the estimates (3.6) and (3.7) to estimates of the resolvent.

THEOREM 4.1. The difference approximation is stable with respect to Definition 3.2 if and only if there are constants $\alpha_{0} \geqq 0, K_{1}>0$ such that (4.1), (4.2) have, for every $z$ with $|z|>e^{\alpha_{0} k}$ and every $\tilde{F}^{\wedge} \in l_{2}(x)$, a unique solution with

$$
\left(\frac{|z|-e^{\alpha_{0} k}}{|z|}\right)^{2}\|\hat{w}\|_{x}^{2} \leqq K_{1}^{2}\left(\left\|\tilde{F}^{\wedge}\right\|_{x}^{2}+h \sum_{\mu=-r+1}^{0}\left|g_{\mu}\right|^{2}\right) .
$$

Proof. Consider (1.5)-(1.7) with $f(\sigma k) \equiv 0$ and assume that the approximation is stable in the sense of Definition 3.2. Introduce $w=e^{-\alpha t} v$ as a new variable. Then, $w$ is the solution of

$$
\begin{gathered}
Q_{-1} w_{\nu}(t+k)=\sum_{\sigma=0}^{s} e^{-\alpha(\sigma+1) k} Q_{\sigma} w_{\nu}(t-\sigma k)+k \tilde{F}_{\nu}(t), \\
w_{\mu}(t+k)-\sum_{\sigma=0}^{s} e^{-\alpha(\sigma+1) k} S_{\sigma}^{(\mu)} w_{1}(t-\sigma k)=\tilde{g}_{\mu}(t)
\end{gathered}
$$

with

$$
w(\sigma k) \equiv 0, \quad \sigma=0,1, \cdots, s,
$$

and

$$
\tilde{F}_{\nu}=e^{-\alpha(t+k)} F_{\nu}, \quad \tilde{g}_{\mu}=e^{-\alpha(t+k)} g_{\mu} .
$$

The stability assumption means that there is an estimate (3.6) so that

$$
\left(\frac{\alpha-\alpha_{0}}{\alpha k+1}\right)^{2}\|w\|_{x, t}^{2} \leqq K_{0}^{2}\left(\|\tilde{F}\|_{x, t}^{2}+h^{-1} \sum_{\mu=-r+1}^{0}\left\|\tilde{g}_{\mu}\right\|_{t}^{2}\right)
$$

The starting time $t=0$ is completely arbitrary. We can choose any time $t=t_{0}=\tau_{0} k^{\prime}$ i.e., replace (4.6) by $w\left(t_{0}+\sigma k\right)=0$ and study the difference approximation for $t \geqq t_{0}$ instead. Defining $\tilde{F} \equiv w \equiv 0$ for $t \leqq t_{0}$, we consider the Eqs. (4.4), (4.5) for all $t=\tau k, \tau=0, \pm 1, \pm 2, \cdots$. By assumption, there is for every $\tilde{F}$ and $\tilde{g}$ with compact support a solution for which (4.7) holds, provided we replace $\|\tilde{F}\|_{x, t},\left\|\tilde{g}_{\mu}\right\|_{t}$ by the corresponding sums over all $t=\tau k, \tau=0, \pm 1, \pm 2, \cdots$. Therefore, we can Fourier transform (4.4), (4.5) with respect to $t$. With $\omega$ denoting the (real) variable dual to $t$ and using the notation $z=e^{(\alpha+i \omega) k}$, we get the resolvent Eqs. (4.1), (4.2), where $\hat{w}, \tilde{F}^{\wedge}$, and $\tilde{g}_{\mu}$ are the Fourier transforms of $w, k \tilde{F}$ and $\tilde{g}_{\mu}$ respectively. (The choice of notation $\tilde{F}^{\wedge}$ rather than $k \tilde{F}^{\wedge}$ to denote the transform of $k \tilde{F}$ may seem strange but simplifies the presentation.)

By Parseval's relation, the estimate (4.7) goes over into

$$
\left(\frac{\alpha-\alpha_{0}}{\alpha k+1}\right)^{2}\|\hat{w}\|_{x}^{2} \leqq K_{0}^{2} k^{-2}\left(\|\tilde{F}\|_{x}^{2}+\lambda k \sum_{\mu=-r+1}^{0}\left|\hat{g_{\mu}}\right|^{2}\right),
$$


where, as usual, $\lambda=k / h=$ const, and (4.3) follows easily. Now, it is well known that the functions $F$ with compact support are dense in $l_{2}(x, t)$, and the corresponding $g_{\mu}$ are dense in $l_{2}(t)$. Therefore, (4.1), (4.2) have, for every $\hat{F} \in l_{2}(x)$, a solution for which (4.3) holds. This solution is unique by Lemma 4.1.

We have thus proved that if an estimate of type (3.6) holds then the resolvent condition (4.3) must be fulfilled. We shall now prove the converse: Consider the Eqs. (1.5)-(1.7) with $f(\sigma k)=0$. Let $F$ and $g_{\mu}$ have compact support and $k$ be fixed. By Assumption 3.1, these equations have a solution with

$$
\sum_{\tau=0}^{\infty} e^{-2 \beta \tau}\|v(\tau k)\|_{x}^{2}<\infty .
$$

Here, $\beta>0$ is some constant. Replacing $\alpha$ by $\beta / k$, we can Fourier transform the Eqs. (4.4), (4.5) with respect to $t$ and get the Eqs. (4.1) and (4.2) with $z=e^{\beta+i \omega k}$ and

$$
\begin{aligned}
\tilde{F}_{\nu} & =k(2 \pi)^{-1 / 2} \int_{-\infty}^{+\infty} e^{-s(t+k)} F_{\nu}(t) d t, \\
g_{\mu} & =(2 \pi)^{-1 / 2} \int_{-\infty}^{+\infty} e^{-s(t+k)} g_{\mu}(t) d t, \quad s=i \omega+\beta / k .
\end{aligned}
$$

Solving these equations, the inverse Fourier transform gives us

$$
v(t)=e^{(\beta / k) t} w(t)=(2 \pi)^{-1 / 2} \int_{-\infty}^{+\infty} e^{s t} \hat{w}(z) d \omega, \quad s=i \omega+\beta / k, z=e^{s} .
$$

The resolvent condition (4.3) implies that $\hat{w}(z)=\hat{w}\left(e^{s}\right)$ is an analytic function of $s$ for $\operatorname{Re} s>\alpha_{0}$. Therefore, we can choose for $\beta$ any positive constant $\beta=\alpha k>\alpha_{0} k$ and (3.6) follows from Parseval's relation

$$
\left\|e^{-\alpha t} v\right\|_{x, t}^{2}=\int_{-\infty}^{+\infty}\|\hat{w}(z)\|_{x}^{2} d \omega
$$

and (4.3). This proves the theorem.

In the same way, we can prove

THEOREM 4.2. The difference approximation is stable with respect to Definition 3.3 if and only if there are constants $\alpha_{0} \geqq 0, K_{1}>0$ such that (4.1), (4.2) have, for every $z$ with $|z|>e^{\alpha_{0} k}$ and all $F, g, a$ unique solution with

$$
\begin{aligned}
k\left(\frac{|z|-e^{\alpha_{0} k}}{|z|}\right) \sum_{\mu=-r+1}^{0}\left|\hat{w}_{\mu}\right|^{2}+ & \left(\frac{|z|-e^{\alpha_{0} k}}{|z|}\right)^{2}|| \hat{w} \|_{x}^{2} \\
& \leqq K_{1}^{2}\left(k\left(\frac{|z|-e^{\alpha_{0} k}}{|z|}\right) \sum_{\mu=-r+1}^{0}\left|\hat{g}_{\mu}\right|^{2}+\left\|\tilde{F}^{-}\right\|_{x}^{2}\right) .
\end{aligned}
$$

We shall now show that the stability definitions are invariant with respect to perturbations of order $k$. (This is well known for Definition 3.1.)

THEOREM 4.3. Assume that the difference approximation (1.5)-(1.7) is stable in any of the above senses. Perturb the approximation by adding to the difference operators $Q_{\sigma}$ and $S_{\sigma}^{(\mu)}$ terms of order $k$. Then, the resulting difference approximation is also stable in the same sense.

Proof. Let the grid function $G$ be defined by $G_{\nu}=\tilde{F}_{\nu}$ for $\nu \geqq 1, G_{\mu}=\hat{g_{\mu}}$ for $\mu \leqq 0$. Then, we can consider the resolvent Eqs. (4.1) and (4.2) and its perturbation as a 
mapping from $l_{2}(x)$ into itself and write them in the form

$$
\left.(S)(z) \hat{w}=G \quad \text { and } \quad(B)(z)+k S_{1}(z)\right) \hat{w}=G, \quad \hat{w}, G \in l_{2}(x) .
$$

Let the approximation be stable in the sense of Definition 3.2 and let $\alpha_{1}$ be a constant with

$$
\frac{|z| k}{|z|-e^{\alpha_{0} k}}\left\|\oiint_{1}\right\|_{x} \leqq \frac{1}{2 K_{1}} \text { for all }|z|>e^{\alpha_{1} k}
$$

Then, we get for these $z$ values

$$
\begin{aligned}
\|\left(\mathfrak{S}+k\left(S_{1}\right)^{-1} \|_{x}\right. & \leqq \|\left(S ^ { - 1 } \| _ { x } \cdot \| \left(I+k \mathfrak{S}_{1}\left(\mathfrak{S}^{-1}\right)^{-1} \|_{x}\right.\right. \\
& \leqq \frac{\left\|S^{-1}\right\|_{x}}{1-k\left\|S_{1}\right\|_{x}\left\|\mathfrak{S}^{-1}\right\|_{x}} \leqq 2\left\|S^{-1}\right\|_{x}
\end{aligned}
$$

and for Definition 3.2 the theorem is proved. If we replace the norm

$$
\|\hat{w}\|_{x} \text { by }\|\hat{w}\|=\left(\sum_{\mu=-r+1}^{0}\left|\hat{w}_{\mu}\right|^{2}+\|\hat{w}\|_{x}^{2}\right)^{1 / 2} \text {, }
$$

the theorem follows for Definition 3.3 in the same way as above. By the same kind of perturbation argument, we get, from Assumption 3.1:

THEOREM 4.4. There is a constant $z_{\infty}$ such that the estimates (4.3) and (4.8) hold for $|z| \geqq\left|z_{\infty}\right|$.

Proof. For $|z| \rightarrow \infty$, the resolvent Eqs. (4.1), (4.2) and the estimates (4.3) and (4.8) converge to the equation of Assumption 3.1 and its estimate.

5. The Main Results. In this section, we want to formulate the main results of this paper. By Theorem 4.3, we can neglect $B$ and assume that the coefficients are independent of $h$. Thus, the resolvent Eqs. (4.1), (4.2) can be written as

$$
\begin{gathered}
\sum_{i=-r}^{p} A_{j}(z) E^{i} \hat{w}_{\nu}=\hat{F_{\nu}}, \quad A_{j}(z)=-\sum_{\sigma=0}^{s} z^{-\sigma-1} A_{i \sigma}+A_{i(-1)}, \\
\hat{w} \in l_{2}(x), \quad \hat{w}_{\mu}=\sum_{i=1}^{q}\left(C_{i}(z) \hat{w}_{j}\right)+\hat{g_{\mu}}, \\
C_{j}(z)=\sum_{\sigma=-1}^{s} z^{-\sigma-1} C_{i \sigma}, \quad \mu=r+1, \cdots, 0 .
\end{gathered}
$$

We shall start with stating the assumptions.

Assumption 5.1. The approximation is stable for the Cauchy problem, i.e., if we consider (1.5) for all $\nu=0, \pm 1, \pm 2, \cdots$, then we have, for $\alpha>\alpha_{0}$,

$$
\left\|e^{-\alpha t} v(t)\right\|^{2} \leqq K_{0}^{2} \sum_{\sigma=0}^{s}\|f(\sigma k)\|^{2}, \quad \text { with }\|f\|^{2} \equiv \sum_{\nu=-\infty}^{\infty}\left|f_{\nu}\right|^{2} h .
$$

Consider the Eqs. (1.5) with constant and of $h$ independent coefficients for the Cauchy problem and Fourier transform them with respect to $x$. If $F=0$, we get

$$
\hat{Q}_{-1}(i \xi) \hat{v}(t+k)=\sum_{\sigma=0}^{s} \hat{Q}_{\sigma}(i \xi) \hat{v}(t-\sigma k), \quad \hat{Q}_{\sigma}=\sum_{j=-r}^{p} A_{i \sigma} e^{i j \xi}
$$


Then, Assumption 5.1 implies:

(1) The von Neumann condition is fulfilled, i.e., the eigenvalue problem

$$
\left(\hat{Q}_{-1}(i \xi)-\sum_{\sigma=0}^{s} \hat{Q}_{\sigma}(i \xi) z^{-\sigma-1}\right) y=0
$$

which may also be written

$$
\sum_{i=-r}^{p} A_{j}(z) e^{i i \xi} y=0, \quad A_{j}(z)=-\sum_{\sigma=0}^{s} z^{-\sigma-1} A_{i \sigma}+A_{j(-1)},
$$

has no solution $z$ with $|z|>1$.

(2) If $z=z_{0}$ is an eigenvalue with multiplicity $q$ and $\left|z_{0}\right|=1$, then there are precisely $q$ corresponding linearly independent eigenvectors.

We always make either

Assumption 5.2. Let $z_{i}, j=1,2, \cdots,(s+1) n$, be the eigenvalues of (5.3). If $z_{i}=z_{i}$ for some $\xi=\xi_{0}$ and $\left|z_{i}\right|=1$, then $z_{i}, z_{i}$ are continuously differentiable and

$$
\partial z_{\nu}\left(\xi_{0}\right) / \partial \xi \neq 0, \quad \nu=i, j
$$

or

Assumption 5.3. The matrices $A_{j \sigma}$ can be transformed to diagonal form by the same transformation.

Furthermore, we assume

Assumption 5.4. The approximation is either (strictly) dissipative or nondissipative, i.e., for the eigenvalues $z_{i}$ of (5.3), we have either $\left|z_{j}\right|<1$ for all $j$ and $0<|\xi| \leqq \pi$ or $\left|z_{j}\right|=1$ for all $j$ and all $\xi$. We do not know of any used difference approximation for which neither Assumption 5.2 nor Assumption 5.3 is fulfilled.

For convenience only, we make also

Assumption 5.5. $A_{p}(z)$ and $A_{-r}(z)$ are nonsingular for $|z| \geqq 1$.

To derive stability conditions for the Definition 3.3, we need only consider (5.1), (5.2) with $\tilde{F}^{\wedge}=0$, i.e. we replace (5.1) by

$$
\sum_{i=-r}^{p} A_{i}(z) E^{i} \hat{w}_{\nu}=0 .
$$

(5.1a) is an ordinary difference equation with constant coefficients. Therefore, its general solution belonging to $l_{2}(x)$ can be written in the form

$$
w_{\nu}=\sum_{\left|\kappa_{i}\right|<1} P_{j}(\nu) \kappa_{j}^{\nu}=\sum_{\left|\kappa_{i}\right|<1} P_{j}(\nu, z)\left(\kappa_{i}(z)\right)^{\nu} .
$$

Here, $\kappa_{i}$ are the solutions of

$$
\operatorname{det}\left|\sum_{j=-r}^{p} A_{j}(z) \kappa^{j}\right|=0,
$$

and $P_{j}(\nu)$ are polynomials in $\nu$ with vector coefficients. The degree of $P_{i}(\nu)$ is one less than the multiplicity of the corresponding $\kappa_{j}$. We shall now show that for $|z|>1$, (5.5) consists of $n r$ linearly independent solutions. We start with

LEMmA 5.1. Let $|z|>1$. Then the Eq. (5.6) has no solution $\kappa$ with $|\kappa|=1$.

Proof. Assume that (5.5) had a solution $\kappa=e^{i \xi}, \xi$ real: Then there is a vector $y \neq 0$ such that 


$$
\sum_{i=-r}^{p} A_{i}(z) e^{i j \xi} y=0 .
$$

This is the same equation as (5.3). The von Neumann condition implies that $|z| \ngtr 1$, a contradiction to the assumption.

The solutions of (5.6) thus split for $|z|>1$ into two groups: $M_{1}$ containing those eigenvalues $\kappa$ with $|\kappa|<1$, and $M_{2}$ containing those with $|\kappa|>1$. Furthermore, the number of eigenvalues belonging to $M_{1}$ or $M_{2}$, counted according to their multiplicity, is independent of $z$ for $|z|>1$. Therefore, these numbers can be determined by considering (5.1), (5.2) for $z \rightarrow \infty$. In the limit $z \rightarrow \infty$, we get the equations

$$
\sum_{j=-r}^{p} A_{i(-1)} E^{i} \hat{w}_{\nu}=Q_{-1} \hat{w}_{\nu}=0
$$

with the $n r$ linearly independent boundary conditions

$$
\hat{w}_{\mu}-\sum_{j=1}^{q} C_{j(-1)} \hat{w}_{i}=\hat{w}_{\mu}-S_{-1}^{(\mu)} \hat{w}_{1}=\hat{g_{\mu}} .
$$

By Assumption 3.1, these equations have, for every set of values $g$, a unique solution belonging to $l_{2}(x)$. Therefore, we have proved

LEMMA 5.2. The number of linearly independent solutions in (5.5) is equal to $n r$.

Thus, the general solution (5.5) depends on $n r$ free parameters $c=\left(c_{1}, \cdots, c_{n r}\right)^{\prime}$. Inserting (5.5) into the boundary conditions (5.2) gives us a linear system of equations

$$
E(z) c=\hat{\tilde{g}}, \quad \hat{g}=\left(\hat{g_{0}}, \cdots, \hat{g_{-r+1}}\right)^{\prime},
$$

and (5.1a), (5.2) have a unique solution if and only if $\operatorname{det}|E(z)| \neq 0$.

We can now formulate our main result.

THEOREM 5.1. The difference approximation is stable in the sense of Definition 3.3 if and only if the Eqs. (5.1a), (5.2) have a unique solution in $l_{2}(x)$ for all $|z|>1$ and there is a constant $K_{2}$, independent of $z$ and $g$, such that

$$
\sum_{\mu=-r+1}^{0}\left|\hat{w}_{\mu}\right|^{2} \leqq K_{2}^{2} \sum_{\mu=-r+1}^{0}\left|\hat{g}_{\mu}\right|^{2}, \quad|z|>1 .
$$

In Lemma 10.3 we shall formulate (5.8) also as a determinant condition. In [1], this was expressed in the following way: There are no eigenvalues or generalized eigenvalues for $|z| \geqq 1$.

For the stability Definition 3.2, we do not get such a simple result and we formulate the conditions first in Section 10.

THEOREM 5.2. If the approximation is dissipative, then it is stable in the sense of Definition 3.2 if and only if the conditions of Theorem 10.3 are fulfilled.

It should be pointed out that the above conditions need only hold in a neighbourhood of $|z|=1$.

THEOREM 5.3. Let $\eta>0$ be any constant and assume that $\operatorname{det}|E(z)| \neq 0$ for $|z|>1+\eta$ and that the above conditions hold for $1<|z|<1+\eta$. Then the approximation is stable.

There is no difficulty to generalize the above theorems to the case with two boundaries. The following theorem is valid for any of the stability definitions.

THEOREM 5.4. Consider the difference approximation for $t \geqq 0$ and $0 \leqq x \leqq 1$ and 
assume that the corresponding left and right quarter-plane problems (which we get by removing one boundary to infinity) are stable. Then the original problem is also stable.

6. Applications. Difference Approximations on a Quarter Space. In this section, we will investigate various boundary conditions for some commonly used approximations.

I. Lax-Wendroff $(L-W)$ Type Dissipative Schemes. Consider the system (1.1), (1.2), (1.3), (1.4) with $F(x, t) \equiv 0, g(t) \equiv 0$ and the difference approximation

$$
v(t+k)=v(t)+k B v(t)+k A D_{0} v(t)+\frac{1}{2} k^{2} C D_{+} D_{-} v(t)
$$

where $C>0$ is a matrix that can be transformed to diagonal form together with $A$ : The condition

$$
v_{0}^{\mathrm{I}}=S v_{0}^{\mathrm{II}}
$$

will always be used, and the following possibilities for specifying $v_{0}^{\mathrm{II}}$ will be studied:

$$
\begin{aligned}
\left(h D_{+}\right)^{i} v_{0}^{\mathrm{II}}=0, \quad j \text { natural number, } & \\
v_{0}^{\mathrm{II}}(t+k) & =v_{0}^{\mathrm{II}}(t)+k A^{\mathrm{II}} D_{+} v_{0}^{\mathrm{II}}(t), \\
v_{0}^{\mathrm{II}}(t+k)+v_{1}^{\mathrm{II}}(t+k) & -k A^{\mathrm{II}} D_{+} v_{0}^{\mathrm{II}}(t+k) \\
& =v_{0}^{\mathrm{II}}(t)+v_{1}^{\mathrm{II}}(t)+k A^{\mathrm{II}} D_{+} v_{0}^{\mathrm{II}}(t) .
\end{aligned}
$$

The resolvent equation for (6.1) is in scalar form

(6.4) $z \hat{w}_{\nu}=\hat{w}_{\nu}+\frac{\lambda a}{2}\left(\hat{w}_{\nu+1}-\hat{w}_{\nu-1}\right)+\frac{\lambda^{2} c}{2}\left(\hat{w}_{\nu+1}-2 \hat{w}_{\nu}+\hat{w}_{\nu-1}\right), \quad \nu=1,2, \cdots$,

where, by Theorem 4.3 , we have neglected the term $k B \hat{w}_{\nu}$.

The characteristic equation takes the form

$$
z \kappa=\kappa+\frac{\lambda a}{2}\left(\kappa^{2}-1\right)+\frac{\lambda^{2} c}{2}(\kappa-1)^{2} .
$$

The following lemma is proved in [3].

LEMMA 6.1. There exists a $\delta>0$, such that for the roots $\kappa_{1}, \kappa_{2}$ :

I. If $a<0$, then

$$
\begin{array}{rlrl}
\left|\kappa_{1}\right| & <1 & & \text { for }|z| \geqq 1, z \neq 1, \\
\kappa_{1} & =1 & & \text { for } z=1, \\
\left|\kappa_{2}\right| \geqq 1+\delta & & \text { for }|z| \geqq 1 .
\end{array}
$$

II. If $a>0$, then

$$
\begin{aligned}
\left|\kappa_{1}\right| \leqq 1-\delta & \text { for }|z| \geqq 1, \\
\left|\kappa_{2}\right|>1 & \text { for }|z| \geqq 1, z \neq 1, \\
\kappa_{2}=1 & \text { for } z=1 .
\end{aligned}
$$

With help of this lemma, we can prove

THEOREM 6.1. The approximation (6.1) is stable in the sense of Definition 3.3 with 
the boundary condition (6.2) in combination with any one of the conditions (6.3a), (6.3b), (6.3c).

Proof. With this form of boundary conditions, it is clearly sufficient to show (5.8) for each scalar equation with $a>0$. The general solution of (6.4) belonging to $l_{2}(x)$ has the form

$$
\hat{w}_{\nu}=\kappa_{1}^{\nu} \hat{w}_{0}, \quad\left|\kappa_{1}\right| \leqq 1-\delta .
$$

We insert this solution into the condition $\left(h D_{+}\right)^{i} \hat{w}_{0}^{\text {II }}=0$, corresponding to (6.3a), and obtain

$$
\hat{w}_{0}\left(\kappa_{1}-1\right)^{j}=0 .
$$

Since $\left|\kappa_{1}-1\right| \geqq \delta$ for $|z| \geqq 1$, the determinant condition of (10.3) mentioned in Theorem 5.1 is satisfied and stability follows. (This was already shown in [3].)

(6.3b) gives the condition

$$
\hat{w}_{0}\left(1-\kappa_{1} \frac{\lambda a}{z-1+\lambda a}\right)=0
$$

and for $\lambda a<1$ (stability condition for the Cauchy-problem), we have

$$
\left|1-\kappa_{1} \frac{\lambda a}{z-1+\lambda a}\right|=\frac{\left|z-1+\lambda a-\kappa_{1} \lambda a\right|}{|z-1+\lambda a|} \geqq \delta \quad \text { for }|z| \geqq 1 \text {; }
$$

stability. (6.3c) implies

$$
\hat{w}_{0}\left(1+\kappa_{1} \frac{z-1-\lambda a(z+1)}{z-1+\lambda a(z+1)}\right)=0 .
$$

However,

$$
\left|1+\kappa_{1} \frac{z-1-\lambda a(z+1)}{z-1+\lambda a(z+1)}\right| \geqq 1-\left|\kappa_{1}\right| \cdot\left|\frac{z-1-\lambda a(z+1)}{z-1+\lambda a(z+1)}\right|>\delta,
$$

since $\operatorname{Re}(z+1) /(z-1) \geqq 0$ for $|z| \geqq 1$; stability, and the proof of the theorem is completed.

II. Nondissipative Schemes: Leap-Frog $(L-F)$ and Crank-Nicolson $(C-N)$. Consider the leap-frog approximation

$$
v(t+k)=2 k A D_{0} v(t)+v(t-k)+k B(v(t+k)+v(t-k))
$$

with resolvent equations, for $B=0$,

$$
z^{2} \hat{w}_{\nu}=z \lambda a\left(\hat{w}_{\nu+1}-\hat{w}_{\nu-1}\right)+\hat{w}_{\nu}, \quad \nu=1,2, \cdots,
$$

and characteristic equation

$$
\kappa^{2}-\frac{z^{2}-1}{\lambda a z} \kappa-1=0 .
$$

For $z=(1+\eta) e^{i \theta},(6.9)$ has the roots

$$
\kappa_{1,2}=\frac{i \sin \theta+\eta\left(1+\frac{1}{2} \eta\right) e^{i \theta}}{\lambda a(1+\eta)} \pm\left(1-\frac{\left(\sin \theta-i \eta\left(1+\frac{1}{2} \eta\right) e^{i \theta}\right)^{2}}{(\lambda a(1+\eta))^{2}}\right)^{1 / 2}
$$

For $z=e^{i \theta}$, we thus get 


$$
\kappa_{1}=i \frac{\sin \theta}{\lambda a} \pm\left(1-\frac{\sin ^{2} \theta}{(\lambda a)^{2}}\right)^{1 / 2}=\frac{\lambda a \pm\left((\lambda a)^{2}-\sin ^{2} \theta\right)^{1 / 2}+i \sin \theta}{\lambda a \pm\left((\lambda a)^{2}-\sin ^{2} \theta\right)^{1 / 2}-i \sin \theta}
$$

where the sign is to be chosen opposite to that of $\cos \theta$.

LEMMA 6.2. The roots of (6.9) have the following properties:

If $|z|>1$, then $\left|\kappa_{1}\right|<1,\left|\kappa_{2}\right|>1$ and $\operatorname{Re} \kappa_{1} \leqq 0$ for $\operatorname{Re} z>0, \operatorname{Re} \kappa_{1} \geqq 0$ for $\operatorname{Re} z<0$.

If $z=e^{i \theta}$, then

$$
\begin{aligned}
& \left|\kappa_{1}\right|<1, \quad\left|\kappa_{2}\right|>1 \text { for }|\sin \theta|>\lambda a, \\
& \left|\kappa_{1}\right|=1, \quad\left|\kappa_{2}\right|=1 \quad \text { for }|\sin \theta| \leqq \lambda a \text {, } \\
& \kappa_{1}=-1, \quad \kappa_{2}=1 \quad \text { for } \theta=0 \text {, } \\
& \kappa_{1}=1, \quad \kappa_{2}=-1 \text { for } \theta=\pi, \\
& \kappa_{1}=\kappa_{2}= \pm i \quad \text { for } \sin \theta= \pm \lambda a \text {. }
\end{aligned}
$$

Let us now again investigate the boundary conditions (6.3), and also

$$
\left({ }^{-} \Delta_{+}\right)^{i} v_{0}^{\mathrm{II}}=0
$$

with ${ }^{-} \Delta_{+}$defined by

$$
{ }^{-} \Delta_{+} u_{\nu}(t) \equiv u_{\nu+1}(t-k)-u_{\nu}(t) .
$$

THEOREM 6.2. The approximation (6.7) is stable in the sense of Definition 3.3 with any of the boundary conditions (6.3b), (6.3c), (6.11) in combination with (6.2), but not with (6.3a).

Proof. For $|z|>1$, the general solution of (6.8) belonging to $l_{2}(x)$ is $\hat{w}_{\nu}=\hat{w}_{0} \kappa_{1}^{\nu}$, $\left|\kappa_{1}\right|<1$. It follows immediately that there is no nontrivial solution for $|z|>1$. Since $\kappa_{1}(-1)=1$ satisfies $(6.6 \mathrm{a})$, the determinant condition cannot be satisfied and that approximation is not stable in the sense of Definition 3.3. Condition (6.6b) gives $\hat{w}_{0}=0$ for $|z|>1$. For $z=e^{i \theta},\left|\operatorname{Im}\left(z-1-\lambda a\left(\kappa_{1}-1\right)\right)\right|=\left|\sin \theta-\lambda a \operatorname{Im} \kappa_{1}\right|>0$ when $|\sin \theta|>\lambda a$, and for $|\sin \theta| \leqq \lambda a,\left|\operatorname{Re}\left(z-1-\lambda a\left(\kappa_{1}-1\right)\right)\right|>\left(1-\lambda^{2} a^{2}\right)^{1 / 2}-1+$ $\lambda a>0$ for $\lambda a<1$. Also, $|z-1+\lambda a|<2-\lambda a$, and we have stability.

Condition (6.6c), for $z= \pm 1$, is $2 \hat{w}_{0}=0$ and, for $z=e^{i \theta}, \theta \neq 0, \pi$,

$$
\begin{aligned}
& \left|1+\kappa_{1} \frac{z-1-\lambda a(z+1)}{z-1+\lambda a(z+1)}\right|=\left|1+\kappa_{1} \frac{i \sin \theta-\lambda a(1+\cos \theta)}{i \sin \theta+\lambda a(1+\cos \theta)}\right| \\
& =\left|1-\left(\frac{\lambda a \pm\left(\lambda^{2} a^{2}-\sin ^{2} \theta\right)^{1 / 2}+i \sin \theta}{\lambda a \pm\left(\lambda^{2} a^{2}-\sin ^{2} \theta\right)^{1 / 2}-i \sin \theta}\right)\left(\frac{\lambda a(1+\cos \theta)-i \sin \theta}{\lambda a(1+\cos \theta)+i \sin \theta}\right)\right| \\
& =\left|\frac{2 i \sin \theta\left( \pm\left(\lambda^{2} a^{2}-\sin ^{2} \theta\right)^{1 / 2}-\lambda a \cos \theta\right)}{\left(\lambda a \pm\left(\lambda^{2} a^{2}-\sin ^{2} \theta\right)^{1 / 2}-i \sin \theta\right)(\lambda a(1+\cos \theta)+i \sin \theta)}\right|>0,
\end{aligned}
$$

proving the stability.

Condition (6.11) gives

$$
\hat{w}_{0}\left(1-z^{-1} \kappa_{1}\right)^{i}=0,
$$

but from Lemma 6.2, $\left|1-z^{-1} \kappa_{1}\right|>0$ follows; stability, and the theorem is proved.

We now consider the Crank-Nicolson scheme 


$$
v(t+k)-\frac{1}{2} k A D_{0} v(t+k)=v(t)+\frac{1}{2} k A D_{0} v(t),
$$

with resolvent equation

$$
4(z-1) \hat{w}_{\nu}=\lambda(z+1) a\left(\hat{w}_{\nu+1}-\hat{w}_{\nu-1}\right), \quad \nu=1,2, \cdots,
$$

and characteristic equation

$$
\kappa^{2}-\frac{4}{\lambda a} \frac{z-1}{z+1} \kappa-1=0, \quad z \neq-1 .
$$

For $z=e^{i \theta},(6.15)$ has the roots

$\kappa_{1,2}=\frac{2 i}{\lambda a} \tan \frac{\theta}{2} \pm\left(1-\frac{4 \tan ^{2} \theta / 2}{(\lambda a)^{2}}\right)^{1 / 2}=\frac{\lambda a \pm\left(\lambda^{2} a^{2}-4 \tan ^{2} \theta / 2\right)^{1 / 2}+2 i \tan \theta / 2}{\lambda a \pm\left(\lambda^{2} a^{2}-4 \tan ^{2} \theta / 2\right)^{1 / 2}-2 i \tan \theta / 2}$.

By studying the roots for $z=(1+\eta) e^{i \theta}, \eta \rightarrow 0$, we obtain, for $a>0$,

LEMMA 6.3. The roots of (6.15) have the following properties:

If $|z|>1$, then $\left|\kappa_{1}\right|<1,\left|\kappa_{2}\right|>1$ with $\operatorname{Re} \kappa_{1}<0$.

If $z=e^{i \theta}$, then

$$
\begin{gathered}
\left|\kappa_{1}\right|<1, \quad\left|\kappa_{2}\right|>1 \text { for }\left|\tan \frac{\theta}{2}\right|>\frac{\lambda a}{2}, \\
\kappa_{1}=\frac{2 i}{\lambda a} \tan \frac{\theta}{2}-\left(1-\frac{4 \tan ^{2} \theta / 2}{(\lambda a)^{2}}\right)^{1 / 2} \text { for }\left|\tan \frac{\theta}{2}\right| \leqq \frac{\lambda a}{2} . \\
\kappa_{2}=\frac{2 i}{\lambda a} \tan \frac{\theta}{2}+\left(1-\frac{4 \tan ^{2} \theta / 2}{(\lambda a)^{2}}\right)^{1 / 2}
\end{gathered}
$$

We can now prove

THEOREM 6.3. The approximation (6.13) is stable in the sense of Definition 3.3 with the boundary conditions (6.6a), (6.6b), (6.6c), and (6.11).

Proof. For $|z|>1$, the general solution to (6.14) is $\hat{w}_{\nu}=\hat{w}_{0} \kappa_{1}^{\nu},\left|\kappa_{1}\right|<1$, and again it is clear that the conditions (6.6) imply $\hat{w}_{0}=0$ for $|z|>1$. It is also obvious that we have stability with condition (6.3a) since $\kappa_{1} \neq 1$ always.

For (6.6b), (6.16) gives, for $z=e^{i \theta}$,

$$
\left|1-\kappa_{1} \frac{\lambda a}{z-1+\lambda a}\right|>|1-| \kappa_{1}||>0 \text { for }|\tan \theta / 2|>\lambda a / 2 \text {. }
$$

Also, $\left|\operatorname{Im}\left(z-1-\lambda a\left(\kappa_{1}-1\right)\right)\right| \geqq\left|\sin \theta-\lambda a \operatorname{Im} \kappa_{1}\right|>0$ for $|\tan \theta / 2| \leqq \lambda a / 2$, $\theta \neq 0$, and for $\theta=0, z-1-\lambda a\left(\kappa_{1}-1\right)=2 \lambda a>0$. We thus have stability. For (6.6c), (6.16) gives

$$
\begin{aligned}
\left|1+\kappa_{1} \frac{z-1-\lambda a(z+1)}{z-1+\lambda a(z+1)}\right| & \\
& =\left|1+\frac{\lambda a-\left(\lambda^{2} a^{2}-4 \tan ^{2} \theta / 2\right)^{1 / 2}+2 i \tan \theta / 2}{\lambda a-\left(\lambda^{2} a^{2}-4 \tan ^{2} \theta / 2\right)^{1 / 2}-2 i \tan \theta / 2} \cdot \frac{i \tan \theta / 2-\lambda a}{i \tan \theta / 2+\lambda a}\right| \\
& =\left|\frac{-2 i \tan \theta / 2\left(\lambda a+\left(\lambda^{2} a^{2}-4 \tan ^{2} \theta / 2\right)^{1 / 2}\right)}{\left(\lambda a-\left(\lambda^{2} a^{2}-4 \tan ^{2} \theta / 2\right)^{1 / 2}-2 i \tan \theta / 2\right)(i \tan \theta / 2+\lambda a)}\right|>0
\end{aligned}
$$

for $z=e^{i \theta}$ with $|\tan \theta / 2| \leqq \lambda a / 2, \theta \neq 0$. 
Furthermore, $\left|1+\kappa_{1}(z-1-\lambda a(z+1)) /(z-1+\lambda a(z+1))\right|=2$ for $\theta=0$ and is larger than $|1-| \kappa_{1}||>0$ for $|\tan \theta / 2|>\lambda a / 2$, proving stability. For condition (6.11), $\left|\left(1-z^{-1} \kappa_{1}\right)^{i}\right|>0$ follows easily from (6.16), since $\operatorname{Re} \kappa_{1} \leqq 0$ for $|\tan \theta / 2| \leqq \lambda a / 2$ and $\left|\kappa_{1}\right|<1$ for $|\tan \theta / 2|>\lambda a / 2$.

III. Schemes for Systems with Characteristics in Pairs. For systems of the symmetric type (e.g., the wave equation)

$$
\begin{aligned}
& \frac{\partial}{\partial t} \psi^{(1)}=C \frac{\partial}{\partial x} \psi^{(2)}+F^{(1)}(x, t), \\
& \frac{\partial}{\partial t} \psi^{(2)}=C^{\prime} \frac{\partial}{\partial x} \psi^{(1)}+F^{(2)}(x, t),
\end{aligned}
$$

the use of staggered grids often reduces the computation time (for a given accuracy) by a factor of two. The term 'staggered' means that the vector $\Psi_{\nu}^{(1)}$ is defined at points $x_{\nu}=\nu h$, but the vector $\Psi_{\nu}^{(2)}$ at intermediate points $x_{v+1 / 2}=\left(\nu+\frac{1}{2}\right) h$. With an error of $O\left(h^{2}\right)$, we may then approximate the space derivatives by differences over a single step-length $h:(\partial \psi / \partial x)_{x v} \approx\left(D_{0} \Psi\right)_{x v}=(1 / h)\left(\Psi\left(x_{v+1 / 2}\right)-\Psi\left(x_{v-1 / 2}\right)\right)$. The time-differencing may be chosen as the leap-frog or Crank-Nicolson type.

We shall here investigate the stability of such difference schemes for a pair of scalar equations

$$
\begin{aligned}
& \partial \psi^{(1)} / \partial t=c \partial \psi^{(2)} / \partial x, \\
& \partial \psi^{(2)} / \partial t=c \partial \psi^{(1)} / \partial x,
\end{aligned}
$$

and boundary conditions of type

$$
\psi^{(1)}(0, t)=\frac{1+s}{1-s} \psi^{(2)}(0, t)+g(t) \frac{1}{1-s}
$$

with $-1 \leqq s \leqq 1$.

The leap-frog approximation to (6.18) is

$$
\begin{aligned}
\Psi_{\nu}^{(1)}(t+k) & =\Psi_{\nu}^{(1)}(t-k)+2 \lambda c\left(\Psi_{\nu+1 / 2}^{(2)}(t)-\Psi_{\nu-1 / 2}^{(2)}(t)\right) \\
\Psi_{\nu+1 / 2}^{(2)}(t+k) & =\Psi_{\nu}^{(2)}(t-k)+2 \lambda c\left(\Psi_{\nu+1}^{(1)}(t)-\Psi_{\nu}^{(1)}(t)\right) .
\end{aligned}
$$

To guarantee stability of the Cauchy problem, we assume $2 \lambda c \leqq 1$.

If we now temporarily regard $\Psi^{(2)}$ as defined also at points $x_{\nu}=\nu h$ and $\Psi^{(1)}$ at points $x_{v+1 / 2}=\left(\nu+\frac{1}{2}\right) h$, we obtain the resolvent equations

$$
\left(z^{2}-1\right)\left(\Psi^{(1)} \pm \Psi^{(2)}\right)_{\nu}= \pm 2 z \lambda c\left[\left(\Psi^{(1)} \pm \Psi^{(2)}\right)_{\nu+1 / 2}-\left(\Psi^{(1)} \pm \Psi^{(2)}\right)_{\nu-1 / 2}\right]
$$

for $\nu=0, \frac{1}{2}, 1, \cdots$ with the solution, in $l_{2}(x)$,

$$
\left(\Psi^{(1)}+\Psi^{(2)}\right)_{\nu}=\kappa_{1}^{2 \nu}\left(\Psi^{(1)}+\Psi^{(2)}\right)_{0}, \quad\left(\Psi^{(1)}-\Psi^{(2)}\right)_{\nu}=\left(-\kappa_{1}\right)^{2 v}\left(\Psi^{(1)}-\Psi^{(2)}\right)_{0}
$$

or

$$
\Psi_{\nu}^{(1)}=\Psi_{0}^{(1)} \kappa_{1}^{2 \nu}, \quad \Psi_{\nu+1 / 2}^{(2)}=\Psi_{0}^{(1)} \kappa_{1}^{2 \nu+1}, \quad \nu=0,1,2, \cdots
$$

at the original grid-points. $\kappa_{1}$ is here the root of

$$
\kappa^{2}-1=\left(z-z^{-1}\right) \kappa / 2 \lambda c
$$


which satisfies $|\kappa|<1$ for $|z|>1$, cf. (6.9), (6.10) with $a=2 \mathrm{c}$. We must now formulate a boundary condition corresponding to (6.19) in terms of the staggered-grid variables.

For many physical problems, $s=-1$ so that $\Psi^{(1)}(0, t)=\frac{1}{2} g(t)$, and for the resolvent equation, Theorem 5.1 immediately gives stability in the sense of Definition 3.3; no additional condition is required. For $-1<s<1$, we propose the following equation to compute $\Psi_{0}^{(1)}$, the accuracy of which is one order lower than (6.20):

$$
\begin{aligned}
\Psi_{0}^{(1)}(t+k)= & \Psi_{0}^{(1)}(t-k) \\
& +4 \lambda c\left(\Psi_{1 / 2}^{(2)}(t)-\frac{1-s}{1+s} \frac{\Psi_{0}^{(1)}(t+k)+\Psi_{0}^{(1)}(t-k)}{2}+\frac{g(t)}{1+s}\right) .
\end{aligned}
$$

For the resolvent equation, it gives

$$
\left(z-z^{-1}+2 \lambda c \frac{1-s}{1+s}\left(z+z^{-1}\right)\right) \hat{\Psi}_{0}^{(1)}=4 \lambda c \hat{\Psi}_{1 / 2}^{(2)} .
$$

Inserting $\hat{\Psi}_{1 / 2}^{(2)}=\kappa_{1} \hat{\Psi}_{0}^{(1)}$ and using $z-z^{-1}=2 \lambda c\left(\kappa_{1}+\kappa_{2}\right)$, we obtain

$$
\hat{\Psi}_{0}^{(1)}\left(\frac{1-s}{1+s}\left(z+z^{-1}\right)-\kappa_{1}+\kappa_{2}\right)=0 .
$$

Since $\operatorname{Re}\left(\kappa_{1}-\kappa_{2}\right)$ has the opposite sign of $\operatorname{Re}\left(z+z^{-1}\right)$ for $|z| \geqq 1$ and $(1-s) /(1+s) \geqq 0$ for $-1<s<1,\left|(1-s) /(1+s)\left(z+z^{-1}\right)-\kappa_{1}+\kappa_{2}\right|>0$ except maybe for purely imaginary $\kappa_{1}-\kappa_{2}$. In that case, however, $z$ is also purely imaginary, and $\operatorname{Im}\left(\kappa_{1}-\kappa_{2}\right)$ has the opposite sign of $\operatorname{Im}\left(z+z^{-1}\right)$, so that we again have stability according to Definition 3.3. For $s=1$, the boundary condition (6.22) does not give stability in the sense of Definition 3.3, since $\kappa_{1}-\kappa_{2}=0$ for

$$
z=z_{0}= \pm 2 \lambda c i \pm\left(1-4 \lambda^{2} c^{2}\right)^{1 / 2} .
$$

With the energy method, it is, however, easy to show that the approximation is still stable in the sense of Definition 3.2. To obtain the same order of accuracy, we should then change $g(t)$ into $g(t)+2(h / 4 c)^{2} g^{\prime \prime}(t)$.

The Crank-Nicolson scheme

$$
\begin{aligned}
\Psi_{\nu}^{(1)}(t+k)= & \Psi_{\nu}^{(1)}(t) \\
& +\frac{\lambda c}{2}\left(\Psi_{\nu+1 / 2}^{(2)}(t+k)+\Psi_{\nu+1 / 2}^{(2)}(t)-\Psi_{\nu-1 / 2}^{(2)}(t+k)-\Psi_{\nu-1 / 2}^{(2)}(t)\right),
\end{aligned}
$$

$$
\begin{aligned}
\Psi_{\nu+1 / 2}^{(2)}(t+k)= & \Psi_{\nu+1 / 2}^{(2)}(t) \\
& +\frac{\lambda c}{2}\left(\Psi_{\nu+1}^{(1)}(t+k)+\Psi_{\nu+1}^{(1)}(t)-\Psi_{\nu}^{(1)}(t+k)-\Psi_{\nu}^{(1)}(t)\right)
\end{aligned}
$$

gives the resolvent equations

$(6.26)(z-1)\left(\hat{\Psi}^{(1)} \pm \hat{\Psi}^{(2)}\right)_{\nu}= \pm \frac{\lambda c}{2}(z+1)\left[\left(\hat{\Psi}^{(1)} \pm \hat{\Psi}^{(2)}\right)_{\nu+1 / 2}-\left(\hat{\Psi}^{(1)} \pm \hat{\Psi}^{(2)}\right)_{\nu-1 / 2}\right]$ with $\nu=0, \frac{1}{2}, 1, \cdots$. As above, the solution in $l_{2}(x)$ is

$$
\left(\hat{\Psi}^{(1)}+\hat{\Psi}^{(2)}\right)_{\nu}=\kappa_{1}^{2 \nu}\left(\hat{\Psi}^{(1)}+\hat{\Psi}^{(2)}\right)_{0}, \quad\left(\hat{\Psi}^{\mathrm{I}}-\hat{\Psi}^{\mathrm{II}}\right)_{\nu}=\left(-\kappa_{1}\right)^{2 \nu}\left(\hat{\Psi}^{(1)}-\hat{\Psi}^{(2)}\right)_{0},
$$


where $\kappa_{1}$ is now given by (6.15), (6.16) with $a=2 c$, or

$$
\hat{\Psi}_{\nu}^{(1)}=\hat{\Psi}_{0}^{(1)} \kappa_{1}^{2 \nu}, \quad \hat{\Psi}_{\nu+1 / 2}^{(2)}=\hat{\Psi}_{0}^{(1)} \kappa_{1}^{2 \nu+1}, \quad \nu=0,1,2, \cdots .
$$

If $s=-1$ in the boundary condition (6.19), we have directly $\hat{\Psi}_{0}^{(1)}=0$; stability.

For $-1<s<1$, we propose the formula

$\Psi_{0}^{(1)}(t+k)=\Psi_{0}^{(1)}(t)$

$$
\begin{aligned}
+\lambda c\left[\Psi_{1 / 2}^{(2)}(t+k)+\Psi_{1 / 2}^{(2)}(t)-\right. & \frac{1-s}{1+s}\left(\Psi_{0}^{(1)}(t+k)+\Psi_{0}^{(1)}(t)\right) \\
& \left.+\frac{1}{1+s}(g(t+k)+g(t))\right]
\end{aligned}
$$

For the resolvent equation, we then get

$$
\left(z-1+\lambda c \frac{1-s}{1+s}(z+1)\right) \hat{\Psi}_{0}^{(1)}=\lambda c(z+1) \hat{\Psi}_{1 / 2}^{(2)} .
$$

Inserting the $l_{2}(x)$ expressions for $\Psi_{0}^{(1)}$ and $\Psi_{1 / 2}^{(2)}$, this is simplified into

$$
\hat{\Psi}_{0}^{(1)}\left((1-s) /(1+s)-\left(\kappa_{1}-\kappa_{2}\right) / 2\right)=0,
$$

but since (6.15), (6.16) imply $\operatorname{Re} \kappa_{1} \leqq 0, \operatorname{Re} \kappa_{2} \geqq 0,\left|(1-s) /(1+s)-\left(\kappa_{1}-\kappa_{2}\right) / 2\right|>0$; stability.

If $s=1, \kappa_{1}=\kappa_{2}= \pm i$ gives $\left|(1-s) /(1+s)-\left(\kappa_{1}-\kappa_{2}\right) / 2\right|=0$. As for the leap-frog scheme, this leads to stability of type Definition 3.2 but not of type Definition 3.3. Also in this case, we use the conventional energy method [with the norm $\left.\left.\|\Psi\|=(h / 2)\left|\Psi_{0}^{(1)}\right|^{2}+\sum_{\nu=1} h\left|\Psi_{\nu}^{(1)}\right|^{2}+\sum_{\nu=1} h\left|\Psi_{\nu+1 / 2}^{(2)}\right|^{2}\right)^{1 / 2}\right]$.

Finally, we study a "semi-implicit" scheme for a system with characteristics "nearly in pairs":

$$
\begin{aligned}
& \frac{\partial \psi^{(1)}}{\partial t}=-\epsilon \frac{\partial \psi^{(1)}}{\partial x}+c \frac{\partial \psi^{(2)}}{\partial x} \\
& \frac{\partial \psi^{(2)}}{\partial t}=-\epsilon \frac{\partial \psi^{(2)}}{\partial x}+c \frac{\partial \psi^{(1)}}{\partial x}
\end{aligned} \quad \epsilon, c \text { real, }
$$

where $|\epsilon|<|c|$ and the boundary condition is $\Psi^{(1)}(0, t)=\Psi^{(2)}(0, t)+g(t)$. An example of equations of this type are the linearized shallow-water equations, where often $|\epsilon| \ll|c|$. For such cases, the following approximation has been suggested:

$$
\begin{aligned}
& \Psi^{(1)}(t+k)=\Psi^{(1)}(t)-k \epsilon D_{0} \Psi^{(1)}\left(t+\frac{k}{2}\right)+\frac{k c}{2} D_{0}\left(\Psi^{(2)}(t+k)+\Psi^{(2)}(t)\right), \\
& \Psi^{(2)}(t+k)=\Psi^{(2)}(t)-k \epsilon D_{0} \Psi^{(2)}\left(t+\frac{k}{2}\right)+\frac{k c}{2} D_{0}\left(\Psi^{(1)}(t+k)+\Psi^{(1)}(t)\right) .
\end{aligned}
$$

We may then use a staggered grid, in which $\Psi^{(1)}$ is defined at the points $x_{\nu}=\nu h$ and $\Psi^{(2)}$ at the points $x_{v+1 / 2}=\left(\nu+\frac{1}{2}\right) h$ for the integer time levels and the opposite is true for the fractional time levels.

Using the notation $\Psi^{*(1)}(t)$ for $\Psi^{(1)}(t+k / 2)$ and $\Psi^{*(2)}(t)$ for $\Psi^{(2)}(t+k / 2)$, we may write (6.29) as a system of four equations in $\Psi^{(1)}, \Psi^{(2)}, \Psi^{*(1)}$ and $\Psi^{*^{(2)}}$ at times $t$ and $t+k$. The corresponding resolvent equations are 


$$
\begin{aligned}
(z-1)\left(\hat{\Psi}^{(1)}\right. & \left. \pm \hat{\Psi}^{(2)}\right)_{\nu} \\
= & -\lambda \epsilon\left[\left(\hat{\Psi}^{*(1)} \pm \hat{\Psi}^{*(2)}\right)_{\nu+1 / 2}-\left(\hat{\Psi}^{*(1)} \pm \hat{\Psi}^{*(2)}\right)_{\nu-1 / 2}\right] \\
& \pm \frac{\lambda c}{2}(z+1)\left[\left(\hat{\Psi}^{(1)} \pm \hat{\Psi}^{(2)}\right)_{\nu+1 / 2}-\left(\hat{\Psi}^{(1)} \pm \hat{\Psi}^{(2)}\right)_{\nu-1 / 2}\right] \\
(z-1)\left(\hat{\Psi}^{*(1)}\right. & \left. \pm \hat{\Psi}^{*(2)}\right)_{\nu} \\
= & -\lambda \epsilon z\left[\left(\hat{\Psi}^{(1)} \pm \hat{\Psi}^{(2)}\right)_{\nu+1 / 2}-\left(\hat{\Psi}^{(1)} \pm \hat{\Psi}^{(2)}\right)_{\nu-1 / 2}\right] \\
& \pm \frac{\lambda c}{2}(z+1)\left[\left(\hat{\Psi}^{*(1)} \pm \hat{\Psi}^{*(2)}\right)_{\nu+1 / 2}-\left(\hat{\Psi}^{*(1)} \pm \hat{\Psi}^{*(2)}\right)_{\nu-1 / 2}\right]
\end{aligned}
$$

where we have temporarily assumed all variables exist at all points.

The solution in $l_{2}(x)$ is

$$
\begin{aligned}
&\left(\hat{\Psi}^{(1)}+\hat{\Psi}^{(2)}\right)_{\nu}=c_{1} \kappa_{1}^{2 \nu}+c_{2} \kappa_{3}^{2 \nu}, \\
&\left(\hat{\Psi}^{(1)}-\hat{\Psi}^{(2)}\right)_{\nu}=c_{3}\left(-\kappa_{1}\right)^{2 \nu}+c_{4}\left(-\kappa_{3}\right)^{2 \nu}, \\
&\left(\hat{\Psi}^{*(1)}+\hat{\Psi}^{*(2)}\right)_{\nu}=z^{1 / 2}\left(c_{1} \kappa_{1}^{2 \nu}-c_{2} \kappa_{3}^{2 \nu}\right), \\
&\left(\hat{\Psi}^{*(1)}-\hat{\Psi}^{*(2)}\right)_{\nu}=z^{1 / 2}\left(-c_{3}\left(-\kappa_{1}\right)^{2 \nu}+c_{4}\left(-\kappa_{3}\right)^{2 \nu}\right),
\end{aligned}
$$

where

$$
\begin{aligned}
& \kappa_{1,2}^{2}-1=\frac{2(z-1)}{(z+1) \lambda c-2 \lambda \epsilon z^{1 / 2}} \kappa_{1,2}, \\
& \kappa_{3,4}^{2}-1=\frac{2(z-1)}{(z+1) \lambda c+2 \lambda \epsilon z^{1 / 2}} \kappa_{3,4},
\end{aligned}
$$

and $\left|\kappa_{1}\right|,\left|\kappa_{3}\right|<1$ when $|z|>1$. It is easy to show that the Cauchy problem is always stable, since $|\epsilon|<|c|$.

The physical boundary condition $\psi^{(1)}(0, t)=\psi^{(2)}(0, t)+g(t)$ is not used in its original form. Instead, we propose the boundary formula

$$
\begin{gathered}
\Psi_{0}^{(1)}(t+k)=\Psi_{0}^{(1)}(t)-2 \lambda \epsilon\left[\Psi_{1 / 2}^{*(1)}(t)-\frac{1}{2}\left(\Psi_{0}^{(1)}(t+k)+\Psi_{0}^{(1)}(t)\right)\right] \\
+\lambda c\left[\Psi_{1 / 2}^{(2)}(t+k)+\Psi_{1 / 2}^{(2)}(t)-\Psi_{0}^{(1)}(t+k)\right. \\
\left.-\Psi_{0}^{(1)}(t)+g(t+k)+g(t)\right] \\
\Psi_{0}^{*(2)}(t+k)=\Psi_{0}^{*(2)}(t)-2 \lambda \epsilon\left[\Psi_{1 / 2}^{(2)}(t+k)-\frac{1}{2}\left(\Psi_{0}^{*(2)}(t+k)+\Psi_{0}^{*(2)}(t)\right)\right] \\
+\lambda c\left[\Psi_{1 / 2}^{*(1)}(t+k)+\Psi_{1 / 2}^{*(1)}(t)-\Psi_{0}^{*(2)}(t+k)\right. \\
\left.-\Psi_{0}^{*(2)}(t)-2 g(t+k)\right] .
\end{gathered}
$$

For the resolvent equation, they give

$$
\begin{aligned}
& ((z-1)+\lambda(c-\epsilon)(z+1)) \hat{\Psi}_{0}^{(1)}=-2 \lambda \epsilon \hat{\Psi}_{1 / 2}^{*(1)}+\lambda c(z+1) \hat{\Psi}_{1 / 2}^{(2)}, \\
& ((z-1)+\lambda(c-\epsilon)(z+1)) \hat{\Psi}_{0}^{*(2)}=-2 \lambda \epsilon z \hat{\Psi}_{1 / 2}^{(2)}+\lambda c(z+1) \hat{\Psi}_{1 / 2}^{*(1)} .
\end{aligned}
$$

Inserting the $l_{2}(x)$ solution of the resolvent equation, we get

$$
\left(\frac{(c-\epsilon)(z+1)}{c(z+1)-2 \epsilon z^{1 / 2}}-\frac{\kappa_{1}-\kappa_{2}}{2}\right)\left(c_{1}+c_{3}\right)=0,
$$




$$
\left(\frac{(c-\epsilon)(z+1)}{c(z+1)+2 \epsilon z^{1 / 2}}-\frac{\kappa_{3}-\kappa_{4}}{2}\right)\left(c_{2}+c_{4}\right)=0 .
$$

From (6.31) follows that $\operatorname{Re}\left(\kappa_{1}-\kappa_{2}\right) \leqq 0, \operatorname{Re}\left(\kappa_{3}-\kappa_{4}\right) \leqq 0$ for $|z| \geqq 1$, if the condition $|\epsilon| \lambda \leqq 1$ is satisfied and $|\epsilon|<|c|$. Furthermore, $\operatorname{Re}\left((c-\epsilon)(z+1) /\left(c(z+1) \pm 2 \epsilon z^{1 / 2}\right)\right)$ $>0$ if $z \neq-1$ and $\kappa_{1} \neq \kappa_{2}, \kappa_{3} \neq \kappa_{4}$, for $z=-1$ so that we must have $c_{1}+c_{3}=$ $0, c_{2}+c_{4}=0$. Then

$$
\hat{\Psi}_{\nu}^{(1)}=\hat{\Psi}_{\nu+1 / 2}^{(2)}=\hat{\Psi}_{\nu+1 / 2}^{*(1)}=\hat{\Psi}_{\nu}^{*(2)}=0,
$$

proving the stability of the scheme in the sense of Definition 3.3.

7. Further Applications. Difference Approximations on a Half-Strip. In this section, we will consider (1.1), (1.2), (1.3) on the strip $0 \leqq x \leqq 1, t \geqq 0$, with boundary conditions

$$
\begin{aligned}
u^{\mathrm{I}}(0, t) & =S_{0} u^{\mathrm{II}}(0, t)+g_{0}(t), \\
u^{\mathrm{II}}(1, t) & =S_{1} u^{\mathrm{I}}(1, t)+g_{1}(t) .
\end{aligned}
$$

The corresponding conditions for the difference approximation are

$$
\begin{array}{ll}
v_{\mu}(t+k)=\sum_{\sigma=-1}^{s_{1}} S_{0 \sigma}^{(\mu)} v_{1}(t-\sigma k)+g_{0 \mu}(t), & \mu=-r+1, \cdots, 0, \\
v_{\mu}(t+k)=\sum_{\sigma=-1}^{s_{2}} S_{1 \sigma}^{(\mu)} v_{N-1}(t-\sigma k)+g_{1 \mu}(t), & \mu=N, \cdots, N+p-1 .
\end{array}
$$

It is clear from Theorem 5.4 that if the approximation is stable with (7.2a) on $x \geqq 0$, and with (7.2b) on $x \leqq 1$, then it is stable with (7.2a), (7.2b) on $0 \leqq x \leqq 1$. However, there can be an exponential growth of the solution of type $e^{\alpha \tau k}$, since we have the term $\delta h$ in the resolvent estimate. We shall now investigate with which boundary conditions we do, and with which we do not, get such an exponential growth for Eq. (1.1) with $F(x, t) \equiv 0$ and

$$
A=\left(\begin{array}{rr}
-a & 0 \\
0 & a
\end{array}\right) .
$$

As boundary conditions, we take

$$
\begin{aligned}
& u^{\mathrm{I}}(0, t)=u^{\mathrm{II}}(0, t), \\
& u^{\mathrm{I}}(1, t)=u^{\mathrm{II}}(1, t) .
\end{aligned}
$$

The approximation (6.1) with $C=A^{2}$ and boundary conditions,

$$
\begin{array}{cl}
v_{0}^{\mathrm{I}}(t)=v_{0}^{\mathrm{II}}(t), & v_{N}^{\mathrm{I}}(t)=v_{N}^{\mathrm{II}}(t), \\
\left(h D_{+}\right)^{i} v_{0}^{\mathrm{II}}(t)=0, & \left(h D_{-}\right)^{i} v_{N}^{\mathrm{I}}(t)=0,
\end{array}
$$

analogous to (6.3a) can be shown to have exponentially growing solutions for $j=2,3$. However, when using the boundary conditions (7.4b) combined with

$$
\begin{aligned}
\left(1-h^{2} D_{+}^{2}\right) v_{0}^{\mathrm{I}}(t) & =v_{0}^{\mathrm{II}}(t), \\
\left(1-h^{2} D_{-}^{2}\right) v_{N}^{\mathrm{II}}(t) & =v_{N}^{\mathrm{I}}(t),
\end{aligned}
$$

we avoid the exponential growth. 
THEOREM 7.1. The approximation (6.1) with $C=A^{2}$ and boundary conditions (7.4b) and (7.4c) is stable, and has no exponentially growing solutions.

Proof. The stability of both quarter-space problems follows immediately from Lemma 6.1 and the proof of Theorem 6.1. The general solution to (6.4) can be written

$$
\begin{aligned}
\hat{w}_{\nu}^{\mathrm{I}} & =C_{11} \kappa_{1}^{N-\nu}+C_{12} \kappa_{2}^{N-\nu}, \\
\hat{w}_{\nu}^{\mathrm{II}} & =C_{21} \kappa_{1}^{\nu}+C_{22} \kappa_{2}^{\nu},
\end{aligned}
$$

where $\kappa_{1}$ with $\left|\kappa_{1}\right|<1$ and $\kappa_{2}$ with $\left|\kappa_{2}\right| \geqq 1$ are the roots of Eq. (6.5). When inserting the representation (7.5) into the boundary conditions, we obtain

$$
\begin{aligned}
C_{11}\left(\kappa_{1}-1\right)^{i}+C_{12}\left(\kappa_{2}-1\right)^{j}=0, \\
C_{21}\left(\kappa_{1}-1\right)^{i}+C_{22}\left(\kappa_{2}-1\right)^{j}=0, \\
C_{11}\left[\kappa_{1}^{N}-\kappa_{1}^{N-2}\left(\kappa_{1}-1\right)^{2}\right]+C_{12}\left[\kappa_{2}^{N}-\kappa_{2}^{N-2}\left(\kappa_{2}-1\right)^{2}\right]=C_{21}+C_{22}, \\
C_{21}\left[\kappa_{1}^{N}-\kappa_{1}^{N-2}\left(\kappa_{1}-1\right)^{2}\right]+C_{22}\left[\kappa_{2}^{N}-\kappa_{2}^{N-2}\left(\kappa_{2}-1\right)^{2}\right]=C_{11}+C_{12},
\end{aligned}
$$

or

$$
\begin{aligned}
\left(C_{11} \pm C_{21}\right)\left(\kappa_{1}-1\right)^{i}+\left(C_{12} \pm C_{22}\right)\left(\kappa_{2}-1\right)^{i} & =0, \\
\left(C_{11} \pm C_{21}\right)\left(\kappa_{1}^{N}-\kappa_{1}^{N-2}\left(\kappa_{1}-1\right)^{2} \pm 1\right)+\left(C_{12} \pm C_{22}\right)\left(\kappa_{2}^{N}-\kappa_{2}^{N-2}\left(\kappa_{2}-1\right)^{2} \pm 1\right) & =0 .
\end{aligned}
$$

A nontrivial solution may exist if one of the conditions

(7.6) $\left(\kappa_{1}-1\right)^{i}\left[\kappa_{2}^{N}-\kappa_{2}^{N-2}\left(\kappa_{2}-1\right)^{2} \pm 1\right]-\left(\kappa_{2}-1\right)^{i}\left[\kappa_{1}^{N}-\kappa_{1}^{N-2}\left(\kappa_{1}-1\right)^{2} \pm 1\right]=0$ is fulfilled.

Since $\kappa_{1} \kappa_{2}=-(1-\lambda a) /(1+\lambda a),\left|\kappa_{1}\right| \leqq|(1-\lambda a) /(1+\lambda a)|$ and we may neglect terms of order $\kappa_{1}^{N}$. Since also $\left|\kappa_{2}^{2}-\left(\kappa_{2}-1\right)^{2}\right| \geqq 1$, it is sufficient to show that (7.6) cannot be satisfied for $\kappa_{2}=e^{\epsilon+i \xi}, \epsilon>0 ; \epsilon, \xi$ small. Then

$$
\left(\kappa_{1}-1\right)^{i}\left[\kappa_{2}^{N}\left(1-\left(1-\kappa_{2}^{-1}\right)^{2}\right) \pm 1\right]-\left(\kappa_{2}-1\right)^{i}\left[\kappa_{1}^{N}\left(1-\left(1-\kappa_{1}^{-1}\right)^{2}\right) \pm 1\right]
$$

$$
\approx\left(\kappa_{1}-1\right)^{i}\left(1-\left(1-\kappa_{2}^{-1}\right)^{2}\right)\left[(1+\epsilon N) e^{i N \xi} \pm \frac{1-\left(\left(\kappa_{2}-1\right) /\left(\kappa_{1}-1\right)\right)^{i}}{1-\left(1-\kappa_{2}^{-1}\right)^{2}}\right] \text {. }
$$

But

$$
\begin{aligned}
\left|\frac{1-\left(\kappa_{2}-1\right)^{i} /\left(\kappa_{1}-1\right)^{i}}{1-\left(1-\kappa_{2}^{-1}\right)^{2}}\right| & \approx\left|\frac{1-\left(i \xi+\epsilon-\xi^{2} / 2\right)^{i} /(-1)^{i}(1+j \delta)}{1-(\epsilon+i \xi)^{2}}\right| \\
& \leqq 1+\epsilon \text { for } j=1,2,3, \cdots,
\end{aligned}
$$

with $\delta=(1-\lambda a) /(1+\lambda a)$, so that (7.6) can never be satisfied.

We notice also, that, with boundary conditions (7.4a), we would have 1 instead of $1-\left(1-\kappa_{2}^{-1}\right)^{2}$ in the denominator of the last term in (7.7), and, for $j=3,(7.6)$ is satisfied when $\epsilon=3 \xi^{4} /(2 N)$. Equation (6.5) then implies

$$
\left|\kappa_{1}^{2}\right| \approx 1+3 \xi^{4} / N-\delta \xi^{4}
$$

and, hence, the criterion for nongrowing solutions in this case is

$$
N>6 /(1-\alpha) \text {. }
$$

For nondissipative approximations, the investigation analogous to the one above 
becomes more difficult, since we have a neighbourhood of the whole unit circle to take into consideration.

The following table shows those approximations for which there are no exponentially growing eigensolutions. * means that the eigenvalues of $B(z)$ (defined in Section 4) were obtained by a computer program for different $\lambda$-values.

Boundary condition
Approximations with no exponentially growing eigensolutions
(6.3a), $j=1$
$\mathrm{L}-\mathrm{W}$; C-N for $\mathrm{N}$ even
(6.3a), $j=2$
$\mathrm{C}-\mathrm{N}$ for $\mathrm{N}$ even
(6.3a), $j=3$
$\mathrm{L}-\mathrm{W}$ for $\mathrm{N}>6 /(1-\alpha)$; $\mathrm{C}-\mathrm{N}$ for $\mathrm{N}$ even
(6.3b)
L-W; L-F*; C-N*
$(6.3 \mathrm{c})$
L-W for N odd*; L-F*; C-N*
(6.11)
L-F for $\mathrm{N}$ even; $\mathrm{C}-\mathrm{N}$ for $\mathrm{N}$ even

For the wave equation (6.18), the leap-frog scheme (6.20) and the Crank-Nicolson scheme (6.25) have no exponentially growing solutions for the half-strip problem with

$$
\begin{aligned}
& \Psi^{(1)}(0, t)=\frac{1+s}{1-s} \Psi^{(2)}(0, t)+\frac{g_{0}(t)}{1-s}, \\
& \Psi^{(1)}(1, t)=-\frac{1+s}{1-s} \Psi^{(2)}(1, t)+\frac{g_{1}(t)}{1-s},
\end{aligned}
$$

if we use the Eq. (6.22) and (6.27) to compute $\Psi_{0}^{(1)}$ and corresponding formula for $\Psi_{N}^{(1)}, N h=1$. The condition for such an eigensolution to exist is for the leap-frog scheme

$$
\left(\frac{\kappa_{2}}{\kappa_{1}}\right)^{N}= \pm \frac{\left(z+z^{-1}\right)(1-s) /(1+s)+\kappa_{1}-\kappa_{2}}{\left(z+z^{-1}\right)(1-s) /(1+s)+\kappa_{2}-\kappa_{1}} ; \quad \text { (cf. (6.24)) }
$$

and for the Crank-Nicholson scheme

$$
\left(\frac{\kappa_{2}}{\kappa_{1}}\right)^{N}= \pm \frac{2(1-s) /(1+s)+\kappa_{1}-\kappa_{2}}{2(1-s) /(1+s)+\kappa_{2}-\kappa_{1}} ; \quad(\mathrm{cf} .(6.28))
$$

but in both cases, the magnitude of the right-hand side never exceeds 1 while $\left|\kappa_{2}\right|$ is, by definition, never smaller than $\left|\kappa_{1}\right|$. For the modified system (6.28), the analysis is a little more complicated but leads to a condition similar to that for (6.25). No eigensolutions with exponential increase can exist.

8. A Special Form of the Resolvent Equation. In this section, we shall write the resolvent equation (5.1) in a more convenient form. By assumption, $A_{p}(z)$ is nonsingular for $|z| \geqq 1$. Then, we can write (5.1) in the same way as in [1] as a one-step formula:

$$
w_{\nu+1}=M w_{\nu}+G_{\nu}, \quad \nu=1,2, \cdots,
$$

with $w_{\nu}=\left(\hat{w}_{\nu+p-1}, \cdots, \hat{w}_{\nu-r}\right)^{\prime}$ and 


$$
M=-\left(\begin{array}{cccc}
A_{p}^{-1} A_{p-1} & & \cdots & A_{p}^{-1} A_{-r} \\
-I & 0 & \ldots & 0 \\
\vdots & & & \vdots \\
0 & \ldots & -I & 0
\end{array}\right) \quad G_{\nu}=A_{p}^{-1}\left(\begin{array}{c}
\tilde{F}_{\nu}^{\wedge} \\
0 \\
\vdots \\
0
\end{array}\right) .
$$

Using (8.1), we can write the boundary condition (5.2) as $n r$ linear relations for $w_{1}$ only. These we formally write as

$$
S w_{1}=R(G)+g, \quad|R(G)|^{2} \leqq \text { const } \sum_{\nu=1}^{a+1}\left|G_{\nu}\right|^{2}, \quad g=\left(\hat{g_{0}}, \cdots, \hat{g_{-r+1}}\right)^{\prime}
$$

The following lemma is well known.

LEMMA 8.1. The eigenvalues $\kappa$ of $M$ are the solutions of (5.6).

Let us denote by $l_{2}(h)$ the space of all vector functions $w_{\nu}, \nu=1,2, \cdots$, with $\|w\|_{h}^{2}=\sum_{\nu=1}^{\infty}\left|w_{\nu}\right|^{2} h<\infty$. We shall consider (8.1), (8.3) for any $G \in l_{2}(h)$, i.e. not only for the particular $G$ given in (8.2). This introduces no new difficulties because of:

LEMma 8.2. Consider (8.1), (8.3) for any $G \in l_{2}(h)$. The estimate (4.8) holds (with $\alpha_{0}=0$ ) if and only if there is a constant $K_{3}$ such that for all $z$ with $1<|z| \leqq\left|z_{\infty}\right|$ (for the definition of $z_{\infty}$ see Theorem 4.4):

$$
k\left(\frac{|z|-1}{|z|}\right) \sum_{\mu=-r+1}^{0}\left|w_{\mu}\right|^{2}+\left(\frac{|z|-1}{|z|}\right)^{2}\|w\|_{h}^{2} \leqq K_{3}\left(k\left(\frac{|z|-1}{|z|}\right)|g|^{2}+\|G\|_{h}^{2}\right) .
$$

The estimate (4.3) is valid if and only if

$$
\left(\frac{|z|-1}{|z|}\right)^{2}\|w\|_{h}^{2} \leqq K_{3}\left(\|G\|_{h}^{2}+h|g|^{2}\right) .
$$

Proof. Observing that $k / h=\lambda=$ const, it is obvious that (8.4) and (8.5) imply (4.8) and (4.3), respectively.

Assume now that (4.8) or (4.3) holds. Observing that the coefficients of (5.1) and (5.2) are independent of $k$, it follows that also the solutions are independent of $k$ and therefore (4.8) or (4.3) holds with $\alpha_{0}=0$. Write now (8.1) and (8.3) in the form

$$
y_{\nu+1}=M y_{\nu}+G_{\nu}, \quad S y_{1}=L G+g, \quad y=\left(y^{(1)}, \cdots, y^{(p+r)}\right)^{\prime} .
$$

Using the relations

$$
y_{\nu}^{(i+1)}=y_{\nu}^{(i)}+G_{\nu}^{(i)},
$$

we can eliminate $y^{(2)}, \cdots, y^{(p+r)}$ and get the Eqs. (5.1), (5.2) for $y^{(1)}$. Thus, we can estimate $\left\|y^{(1)}\right\|_{h}$ and therefore, by (8.7), also $\left\|y^{(j)}\right\|_{h}$. This proves the lemma.

9. A Normal Form for the Matrix $M$. The aim of this section is to derive a normal form for the matrix $M$ of the one-step formula (8.1). Let us first state the results.

THEOREM 9.1. There exists a transformation $T(z)$ with the following properties:

(1) $T(z), T^{-1}(z)$ are uniformly bounded for $1 \leqq|z| \leqq\left|z_{\infty}\right|$.

(2) $T(z)$ can be chosen to be analytic in the neighbourhood of every point $z_{0}$ with $\left|z_{0}\right| \geqq 1$.

(3) In a neighbourhood of every point $z_{0}$ with $\left|z_{0}\right|>1$, there is a constant $\delta>0$ 
such that

(9.1) $T(z) M(z) T^{-1}(z)=\left(\begin{array}{ll}L & 0 \\ 0 & N\end{array}\right), \quad L^{*} L \leqq(1-\delta) I, N^{*} N \geqq(1+\delta) I$.

(4) In a neighbourhood of every point $z=z_{0}$ with $\left|z_{0}\right|=1$,

$$
T(z) M(z) T^{-1}(z)=\left(\begin{array}{cccccc}
M_{1} & 0 & & \ldots & 0 \\
0 & M_{2} & 0 & \ldots & 0 \\
\vdots & & & & \vdots \\
0 & \ldots & & 0 & M_{l}
\end{array}\right) .
$$

Here,

$$
M_{i}=M_{i}^{(0)}+\left(z-z_{0}\right) M_{i}^{(1)}+\left(z-z_{0}\right)^{2} M_{i}^{(2)}+\cdots,
$$

with

$$
M_{1}=\left(\begin{array}{cc}
L_{1} & 0 \\
0 & N_{1}
\end{array}\right), \quad L_{1}^{*} L_{1} \leqq(1-\delta) I, N^{*} N \geqq(1+\delta) I .
$$

For $j=2, \cdots, l$, the submatrices $M_{i}$ are of order $r_{i} \times r_{i}$ and $M_{i}^{(0)}$ has the form

$$
M_{i}^{(0)}=e^{i \xi_{i}}\left(\begin{array}{cccccc}
1 & 1 & 0 & & \cdots & 0 \\
0 & 1 & 1 & 0 & \cdots & 0 \\
\vdots & & & & & \vdots \\
0 & \cdots & 0 & 1 & 1 \\
0 & \cdots & & 0 & 1
\end{array}\right) \text { for } j=2, \cdots, l^{*}, l^{*}=\text { some integer, }
$$

and

$$
M_{i}^{(0)}=e^{i \xi_{i}}\left(\begin{array}{ccccc}
1 & 0 & & \cdots & 0 \\
0 & 1 & 0 & \cdots & 0 \\
\vdots & & & & \vdots \\
0 & & \cdots & 0 & 1
\end{array}\right) \text { for } j=l^{*}+1, \cdots, l,
$$

with $\xi_{i} \neq \xi_{i}$ for $i \neq j, 2 \leqq i, j \leqq l$.

It should be pointed out that we could replace the Assumptions 5.2 or 5.3 by the assumption that there is a transformation $T(z)$ as described in Theorem 9.1.

The following two theorems contain more information about the blocks $\boldsymbol{M}_{\boldsymbol{i}}$.

THEOREM 9.2. Assume that $M_{j}^{(0)}$ has the form (9.4) and let

$$
M_{j}^{(1)}=\left(\begin{array}{ccc}
m_{11} & \cdots & m_{1 r_{i}} \\
\vdots & & \vdots \\
m_{r_{j} 1} & \cdots & m_{r_{i} r_{j}}
\end{array}\right) e^{i \xi_{j}}
$$

Then, $\operatorname{Re}\left(m_{r_{i} 1} z_{0} \sigma\right)^{1 / r_{i}} \neq 0$ for all complex $\sigma$ with $\operatorname{Re} \sigma>0$ and all definitions of 
$\left(m_{r_{1} 1} z_{0} \sigma\right)^{1 / r_{j}}$. Therefore, the eigenvalues of $M_{i}$ are given by

$$
e^{-i \xi_{i}} \kappa_{j \tau}=1+\left(m_{r j 1}\left(z-z_{0}\right)\right)^{1 / r_{i}}+O\left(z-z_{0}\right)^{2 / r_{i}},
$$

and, if $l_{j}$ denotes the number of $\kappa_{j \tau}$ with $\left|\kappa_{i \tau}\right|<1$ for $|z|>1$, then

$$
l_{i}=\frac{1}{2} r_{i} \quad \text { for } r_{i} \equiv 0(2) \quad \text { or } \quad l_{i}=\frac{1}{2}\left(r_{i} \pm 1\right) \quad \text { for } r_{i} \equiv 1(2) .
$$

THEOREM 9.3. Assume that $M_{i}^{(0)}$ has the form (9.5). Then there is a transformation $T_{j}$, analytic in a neighbourhood of $z=z_{0}$ such that

$$
\begin{aligned}
e^{-i \xi_{i}} T_{i} M_{i} T_{i}^{-1}=I+ & \left(\begin{array}{lllll}
\alpha_{1} & 0 & \ldots & \\
0 & \alpha_{2} & 0 & & \\
\vdots & & & \\
0 & \ldots & 0 & \alpha_{r_{i}}
\end{array}\right)\left(z-z_{0}\right) \\
& +\left(\begin{array}{ccccc}
\beta_{1} & \beta_{12} & \ldots & \beta_{1 r_{i}} \\
0 & \beta_{2} & \ldots & \beta_{2 r_{i}} \\
\vdots & & & \vdots \\
0 & \ldots & & 0 & \beta_{r_{i}}
\end{array}\right)\left(z-z_{0}\right)^{2}+\cdots
\end{aligned}
$$

Here, $\operatorname{Re}\left(2 \beta_{i}-\alpha_{j}\right)^{2} z_{0} \neq 0, \alpha_{i} z_{0}$ is real with

$$
\alpha_{1} z_{0} \leqq \alpha_{2} z_{0} \leqq \cdots \leqq \alpha_{d} z_{0}<0<\alpha_{d+1} z_{0} \leqq \cdots \leqq \alpha_{r_{i}} z_{0}
$$

and

(9.9) $2 \alpha_{\tau} z_{0} c+\alpha_{\tau}^{2} z_{0}^{2}-2 \operatorname{Re} \beta \neq 0$ for all $c>-\frac{1}{2}, \tau=1,2, \cdots, r_{i}$.

Therefore, we can even assume that $M_{i}$ has the form

$$
e^{-i \xi_{i}} M_{i}=\left(\begin{array}{cc}
L_{i} & 0 \\
0 & N_{j}
\end{array}\right)
$$

with

$$
\begin{aligned}
|z|\left(L_{i}^{*} L_{i}-I\right) & \leqq-\delta_{2}(|z|-1) I, \\
|z|\left(N_{j}^{*} N_{i}-I\right) & \geqq \delta_{2}(|z|-1) I, \quad \delta_{2}>0 .
\end{aligned}
$$

Let us now prove these theorems. We start with the following essential lemma.

LEMMA 9.1. There exists a constant $K>0$ such that

$$
\sup _{\varphi}\left|\left(M(z)-e^{i \varphi}\right)^{-1}\right| \leqq K\left|\frac{|z|}{|z|-1}\right|, \quad \varphi \text { real. }
$$

Therefore, if $\kappa$ is an eigenvalue of $M(z)$, then

$$
\inf _{\varphi}\left|\left(\kappa-e^{i \varphi}\right)\right| \geqq K^{-1}\left|\frac{|z|-1}{|z|}\right| .
$$

Proof. Let us consider the Cauchy problem. By assumption, it is stable and therefore, by Theorem 4.1 and Lemma 8.2, the resolvent condition (8.5) is fulfilled, 
i.e. for the solutions of (8.1) considered for all $\nu=0, \pm 1, \pm 2, \cdots$ without boundary conditions, there is an estimate

$$
((|z|-1) /|z|)^{2}\|w\|_{h}^{2} \leqq K_{3}|| G \|_{h}^{2} .
$$

The inequality (9.12) then follows if we Fourier transform (8.1) with respect to $\nu$.

Proof of Theorem 9.1. If $\left|z_{0}\right|>1$, the existence of an analytic transformation $T(z)$, such that (9.3) holds, follows directly from Lemma 5.1 and Lemma 9.1, because the eigenvalues of $M$ are precisely the solutions of (5.6) which split up into the groups $M_{1}$ and $M_{2}$.

If $\left|z_{0}\right|=1$, then it is well known that there is a constant matrix $T\left(z_{0}\right)$ such that

$$
T\left(z_{0}\right) M\left(z_{0}\right) T^{-1}\left(z_{0}\right)=\left(\begin{array}{ccccc}
M_{1}^{(0)} & 0 & & \ldots & 0 \\
0 & M_{2}^{(0)} & 0 & \ldots & 0 \\
\vdots & & & & \vdots \\
0 & & \ldots & & M_{l}^{(0)}
\end{array}\right) .
$$

Now let Assumption 5.2 be fulfilled. If we can show that for the submatrices (9.4) and (9.5) always $e^{i \xi_{\nu}} \neq e^{i \xi_{\mu}}$, then (9.2) follows without difficulties. Assume that $e^{i \xi_{\nu}}=e^{i \xi_{\mu}}=e^{i \xi_{0}}$ for some $z=z_{0}$. Then the equation

$$
\left(M\left(z_{0}\right)-e^{i \xi_{0}} I\right) y=0, \quad y=\left(y^{(1)}, \cdots, y^{(p+r)}\right)^{\prime},
$$

i.e.

$$
\begin{aligned}
A_{p}\left(z_{0}\right) e^{i \xi_{0}} y^{(1)}+\sum_{j=1}^{p+r} A_{p-i}\left(z_{0}\right) y^{(i)} & =0, \\
y^{(i-1)}-e^{i \xi_{0}} y^{(i)} & =0,
\end{aligned}
$$

which can be written as

$$
\begin{gathered}
\left(\hat{Q}_{-1}\left(i \xi_{0}\right)-\sum_{\sigma=0}^{s} \hat{Q}_{\sigma}\left(i \xi_{0}\right) z^{-\sigma-1}\right) y^{(1)}=0, \\
y^{(i-1)}=e^{i \xi_{0}} y^{(i)}, \quad j=2,3, \cdots, p+r,
\end{gathered}
$$

has at least two linearly independent solutions. Therefore, $z_{0}$ must be a solution of

$$
\operatorname{det}\left|\hat{Q}_{-1}\left(i \xi_{0}\right)-\sum_{\sigma=0}^{s} \hat{Q}_{\sigma}\left(i \xi_{0}\right) z_{0}^{-\sigma-1}\right|=0
$$

with multiplicity two at least. Consider now the equation

$$
\operatorname{det}\left|\hat{Q}_{-1}(i \xi)-\sum_{\sigma=0}^{s} \hat{Q}_{\sigma}(i \xi) z^{-\sigma-1}\right|=0
$$

in a neighborhood of $\xi=\xi_{0}$. By Assumption 5.2 for every solution $z_{\nu}(\xi)$ with $z_{\nu}\left(\xi_{0}\right)=$ $z_{0}$, the relation (5.4) holds. Therefore, we can also consider $\kappa=e^{i \xi}$ as a function of $z$ and get, for every eigenvalue $\kappa_{\nu}(z)$ of $M$ with $\kappa_{\nu}\left(z_{0}\right)=e^{i \xi_{0}}$, an expansion

$$
\kappa_{\nu}(z)=e^{i \xi_{0}}+\left(z-z_{0}\right) \partial \kappa_{\nu}\left(z_{0}\right) / \partial z+O\left(z-z_{0}\right)^{1+1 / r},
$$

where $\partial \kappa_{\nu}\left(z_{0}\right) / \partial z=\left(\partial z_{\nu}\left(\xi_{0}\right) / \partial e^{i \xi}\right)^{-1}$. Furthermore, the number of linearly independent 
eigenvectors is equal to the multiplicity of $\kappa_{\nu}\left(z_{0}\right)$. This follows from the stability of the Cauchy problem as explained in the beginning of Section 5. But then we could transform the matrix

$$
\left(\begin{array}{cc}
M_{\mu} & 0 \\
0 & M_{\nu}
\end{array}\right)
$$

to diagonal form which is a contradiction. This proves Theorem 9.1 if the Assumption 5.2 is fulfilled.

If Assumption 5.3 holds, then we can assume that the $A_{j \sigma}$ have diagonal form and get a scalar equation for every component. If the $A_{i \sigma}$ 's in (8.2) are scalars, then it is well known that the Jordan form of $M$ has only one submatrix of type (9.4) associated with each distinct eigenvalue, and the theorem is proved.

Proof of Theorem 9.2. Without restriction, we may assume that $\xi_{\nu}=0$ and $z_{0}=1$. Let $\left(M_{i}-I\right)_{i k}^{-1}, 0<i, k \leqq r=r_{i}$, denote the elements of $\left(M_{i}-I\right)^{-1}$. By Cramer's rule,

$$
\sup _{i, k}\left|(M-I)_{i k}^{-1}\right|=\left|\frac{1+O(z-1)}{\operatorname{det}\left(M_{j}-I\right)}\right|=\left|\frac{1+O(z-1)}{(z-1)\left(m_{r 1}+O(z-1)\right)}\right|,
$$

and therefore, by Lemma 9.1 ,

$$
\left|m_{r 1}\right| \geqq K_{1}>0 .
$$

An easy calculation then shows that the eigenvalues of $M_{j}$ are given by (9.6a). Let now $z=1+\sigma$ and denote by $\left(m_{r 1} \sigma\right)_{\nu}^{1 / r}$ the different values of $\left(m_{r 1} \sigma\right)^{1 / r}$. Let $\varphi=\operatorname{Im}\left(m_{r 1} \sigma\right)_{v}^{1 / r}$, then, by (9.17),

$$
\begin{aligned}
K_{1}(1+O(|z|-1))(|z|-1) & \leqq\left|\operatorname{det}\left(M_{i}-e^{i \varphi}\right)\right|=\left|\prod_{\tau=1}^{r}\left(\kappa_{j \tau}-e^{i \varphi}\right)\right| \\
& =\left|\prod_{\tau=1}^{r}\left(\left(m_{r 1} \sigma\right)_{\tau}^{1 / r}-i \operatorname{Im}\left(m_{r 1} \sigma\right)_{\nu}^{1 / r}+O\left(\sigma^{2 / r}\right)\right)\right| .
\end{aligned}
$$

Therefore, $\operatorname{Re}\left(m_{r 1} \sigma\right)_{i}^{1 / r} \neq 0$ for all $\sigma$ with $\operatorname{Re} \sigma>0$ and (9.6b) follows by an easy calculation.

Proof of Theorem 9.3. Without restriction, we can assume that $\xi_{\nu}=1, z_{0}=1$, that the eigenvalues of $M_{i}^{(1)}$ are all equal and that $M_{i}^{(1)}$ has upper triangular form, i.e.

$$
M_{j}=\left(1+\alpha(z-1) I+(z-1) D+(z-1)^{2} E+\cdots\right)
$$

with

$$
D=\left(\begin{array}{cccccc}
0 & d_{12} & & \cdots & & d_{1 r} \\
0 & 0 & d_{23} & \cdots & & d_{2 r} \\
\vdots & & & & & \vdots \\
0 & & \ldots & & 0 & 0
\end{array}\right) .
$$

We want to show that $\alpha$ is real and different from zero. The eigenvalues of $M_{j \nu}$ have the form 


$$
\kappa_{i r}=1+\alpha(z-1)+O(z-1)^{1+1 / r} .
$$

Let $z=1+x+i y, \alpha=\alpha_{1}+i \gamma$ and $\varphi=\alpha_{1} y+\gamma x ; x, y, \alpha_{1}, \gamma$ real. Then

$$
\left|\kappa_{j \tau}-e^{i \varphi}\right|=\left|\alpha_{1} x-\gamma y\right|+O\left((|x|+|y|)^{1+1 / r}\right),
$$

and Lemma 9.1 implies that $\alpha$ is real and different from zero. Now let $x=y^{1+1 / r}$, $y>0$ and $\varphi=\alpha y$. We then get, from (9.18),

$$
\begin{aligned}
\left|\left(M_{i}-e^{i \varphi} I\right)^{-1}\right| & =\left|\left(\alpha y^{1+1 / r}\left(I+\frac{y^{1 / r}+i}{\alpha y^{1 / r}} D\right)\left(I+O\left(y^{1-1 / r}\right)\right)\right)^{-1}\right| \\
= & \left|\alpha^{-1} y^{-(1+1 / r)}\left(I+O\left(y^{1-1 / r}\right)\right) \sum_{\nu=0}^{r-1}\left(-\left(y^{1 / r}+i\right) \alpha^{-1} y^{-1 / r} D\right)^{\nu}\right| .
\end{aligned}
$$

But Lemma 9.1 implies

$$
\left|\left(M_{j}-e^{i \varphi} I\right)^{-1}\right| \leqq K_{1}(|z|-1)^{-1} \leqq K_{2} y^{-(1+1 / r)},
$$

and, therefore, (9.19) is possible only if $D \equiv 0$. We have thus proved that $(9.7)$ and (9.8) hold. Therefore, we get for the eigenvalues

$$
\kappa_{i \tau}=1+\alpha_{\tau}(z-1)+\beta_{\tau}(z-1)^{2}+O(z-1)^{2+1 / r} .
$$

Let

$$
z=1+x+i y, \quad \varphi=\alpha_{\tau} y+2 x y \operatorname{Re} \beta_{\tau}+\left(x^{2}-y^{2}\right) \operatorname{Im} \beta_{\tau},
$$

then Lemma 9.1 gives us, for all sufficiently small $x, y$,

$$
\begin{aligned}
\frac{1}{2} K^{-1}\left(|x|+|y|^{2}\right) \leqq \mid \alpha_{\tau} x+x^{2} \operatorname{Re} \beta_{\tau}+ & y^{2}\left(\alpha_{\tau}^{2} / 2-\operatorname{Re} \beta_{\tau}\right) \\
& -2 x y \operatorname{Im} \beta_{\tau}+O(|x|+|y|)^{2+1 / r} \mid,
\end{aligned}
$$

and therefore $\operatorname{Re}\left(\alpha_{\tau}^{2}-2 \operatorname{Re} \beta_{\tau}\right) \neq 0$.

For $x=c y^{2}, c>-\frac{1}{2}$, we have $|z|>1$,

$$
\left|\kappa_{i \tau}\right|^{2} \approx 1+2 \alpha_{\tau} c y^{2}+\left(\alpha_{\tau}^{2}-2 \operatorname{Re} \beta_{\tau}\right) y^{2} .
$$

Assume now that (9.9) is not valid, i.e. there is a $c_{0}$ such that $2 \alpha_{\tau} c_{0}+\alpha_{\tau}^{2}-2 \operatorname{Re} \beta_{\tau}=0$. Then, for $\alpha_{\tau}<0,-\frac{1}{2}<c<c_{0}$, we would have $\left|\kappa_{j \tau}\right|>1$, contradiction.

Without restriction, we may now assume that $e^{-i \xi_{i}} M_{i}$ has the form (9.10) with

$$
L_{i}=I+\left(\begin{array}{llllll}
\alpha_{1} & 0 & & \cdots & & 0 \\
0 & \alpha_{2} & 0 & \cdots & & 0 \\
\vdots & & & & \vdots \\
0 & & \ldots & & 0 & \alpha_{d}
\end{array}\right)\left(z-z_{0}\right)
$$

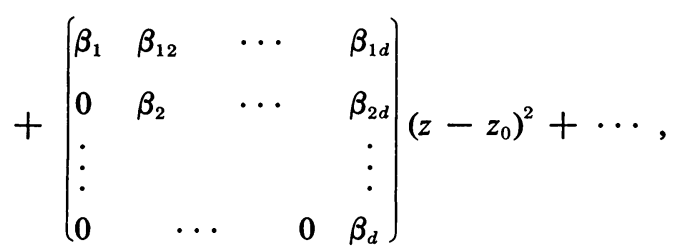




$$
\begin{aligned}
& N_{i}=I+\left(\begin{array}{cccccc}
\alpha_{d+1} & 0 & & \cdots & & 0 \\
0 & \alpha_{d+2} & 0 & \cdots & 0 \\
\vdots & & & & & \vdots \\
0 & & \cdots & & 0 & \alpha_{r_{j}}
\end{array}\right)\left(z-z_{0}\right)
\end{aligned}
$$

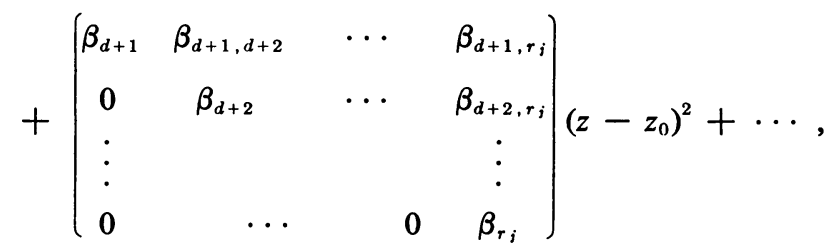

where $\left|\beta_{i j}\right|$ are as small as we like. Then (9.11) follows by an easy calculation from (9.8) and (9.9).

10. Necessary Stability Conditions. We again consider equations with constant coefficients and want to show that the conditions of Theorem 5.1 are necessary for stability.

LEMMA 10.1 (RYABENKII-GodunOv). A necessary condition for stability (in any sense) is that the homogeneous Eqs. (8.1), (8.3) have no nontrivial solution for $|z|>1$.

Proof. Follows directly from (8.4) or (8.5).

For later purposes, we shall derive an explicit algebraic criterion: Let $z$ with $|z|>1$ be fixed and consider the Eqs. (8.1), (8.3). Let $T(z)$ be the transformation in Theorem 9.1 and introduce $y=T(z) w$ as a new variable. Then, (8.1) gets the form

$$
y_{\nu+1}=\left(\begin{array}{l}
y_{\nu+1}^{\mathrm{I}} \\
y_{\nu+1}^{\mathrm{II}}
\end{array}\right)=\left(\begin{array}{ll}
L & 0 \\
0 & N
\end{array}\right)\left(\begin{array}{l}
y_{\nu}^{\mathrm{I}} \\
y_{\nu}^{\mathrm{II}}
\end{array}\right)+T G_{\nu},
$$

and the boundary conditions (8.3) can be written as

$$
D^{\mathrm{I}}(z) y_{1}^{\mathrm{I}}+D^{\mathrm{II}}(z) y_{1}^{\mathrm{II}}=g+R(G) .
$$

If $g \equiv G \equiv 0$, then the general solution of $(10.1)$ which belongs to $l_{2}(h)$ is given by $y_{v}^{\mathrm{I}}=L^{\nu-1} y_{1}^{\mathrm{I}}, y_{\nu}^{\mathrm{II}} \equiv 0$ and we get at once

LEMMA 10.2. The homogeneous Eqs. (8.1), (8.3) have no nontrivial solution if and only if

$$
\operatorname{det}\left|D^{\mathrm{I}}(z)\right| \neq 0 .
$$

Let $z=z_{0}$ with $\left|z_{0}\right|=1$ be fixed and consider the homogeneous Eq. (8.1) with inhomogeneous boundary conditions (8.3). Let $T(z)$ be the transformation of Theorem 9.1 and introduce again $y=T w$ as a new variable. Then, (8.1) gets the form

$$
y_{\nu+1}=\left(\begin{array}{cccc}
M_{1} & 0 & \cdots & 0 \\
0 & M_{2} & & 0 \\
\vdots & & & \\
0 & \ldots & M_{l}
\end{array}\right) y_{\nu},
$$

which, in partitioned form, can be written as 


$$
y_{\nu+1}^{(i)}=M_{i} y_{\nu}^{(i)}, \quad y_{\nu}^{(j)}=\left(y_{\nu 1}^{(i)}, \cdots, y_{\nu r_{j}}^{(i)}\right)^{\prime} .
$$

By (9.1) and (9.10), we can assume that $M_{i}$ for $j=1$ and $j=l^{*}+1, \cdots, l$, have the form

$$
M_{j}=e^{i \xi_{i}}\left(\begin{array}{cc}
L_{i} & 0 \\
0 & N_{j}
\end{array}\right),
$$

$L_{i}, N_{i}$ having the properties defined in (9.3) and (9.11). Therefore, we can write (10.5) as

$$
y_{\nu+1}^{(i 1)}=e^{i \xi_{i}} L_{j} y_{\nu}^{(i 1)}, \quad y_{\nu+1}^{(i 2)}=e^{i \xi_{i}} N_{i} y_{\nu}^{(i 2)}, \quad y^{(i)}=\left(y^{(i 1)}, y^{(j 2)}\right)^{\prime} .
$$

The other $M_{i}, j=2, \cdots, l^{*}$, have, by (9.6a), (9.6b), $l_{j}$ eigenvalues $\kappa_{i \tau}$ with $\left|\kappa_{j \tau}\right|<1$. Correspondingly, we partition the vectors $y^{(i)}, j=2, \cdots, l^{*}$, in the following way:

$$
y_{\nu}^{(j 1)}=\left(y_{\nu 1}^{(j)}, \cdots, y_{\nu l_{j}}^{(j)}\right)^{\prime}, \quad y_{\nu}^{(j 2)}=\left(y_{\nu, l_{j+1}}^{(j)}, \cdots, y_{\nu r_{j}}^{(j)}\right)^{\prime}
$$

and introduce the following notation:

$$
y^{\mathrm{I}}=\left(y^{(11)}, \cdots, y^{(l 1)}\right)^{\prime}, \quad y^{\mathrm{II}}=\left(y^{(12)}, \cdots, y^{(l 2)}\right)^{\prime} .
$$

Then, we can write the boundary conditions again in the form (10.2) and the following theorem holds.

LEMMA 10.3. If the approximation is stable according to Definition 3.3, then the determinant condition (10.3) holds for all $|z| \geqq 1$.

In Sections 12 and 13, we shall prove that this condition is also sufficient for stability.

Proof. For $j=2, \cdots, l^{*}$, it follows, from (9.6a), (9.6b), that there is a nonsingular matrix

$$
U_{j}=I+\left(z-z_{0}\right)^{1 / r_{i}} U_{j}^{(1)}+\cdots
$$

such that

$$
U_{i} M_{i} U_{i}^{-1}=\left(\begin{array}{cccccc}
\kappa_{i 1} & 1 & 0 & \cdots & 0 \\
0 & \kappa_{i 2} & 1 & 0 & \cdots & 0 \\
\vdots & & & & \vdots \\
0 & & \cdots & & 0 & \kappa_{i r_{j}}
\end{array}\right)
$$

with $\left|\kappa_{i 1}\right| \leqq\left|\kappa_{i 2}\right| \leqq \cdots \leqq\left|\kappa_{i l_{i}}\right|<1<\left|\kappa_{i, l_{i+1}}\right| \leqq \cdots \leqq\left|\kappa_{i r_{j}}\right|$ for $|z|>1$. Introducing, instead of $y$,

$$
v=\left(\begin{array}{cccccc}
I & 0 & & \cdots & & 0 \\
0 & U_{2} & 0 & \cdots & 0 \\
\vdots & & & & & \\
0 & \cdots & 0 & U_{l}^{*} & 0 & 0 \\
0 & \cdots & 0 & I & 0 \\
\vdots & & & & & \\
0 & & \cdots & & 0 & I
\end{array}\right) y=\left(I+O\left(\left(z-z_{0}\right)^{1 / r_{j}}\right)\right) y
$$


as a new variable, then the boundary conditions (10.2) get the form $\left(D^{\mathrm{I}}(z)+O\left(z-z_{0}\right)^{1 / r}\right) v_{1}^{\mathrm{I}}+\left(D^{\mathrm{II}}(z)+O\left(z-z_{0}\right)^{1 / r}\right) v_{1}^{\mathrm{II}}=g+R(G), \quad r=\max r_{j}$. If $G \equiv 0$, then $v_{1}^{\mathrm{II}} \equiv 0$ and $v_{1}^{\mathrm{I}}$ is given by

$$
v_{1}^{\mathrm{I}}=\left(D^{\mathrm{I}}(z)+O\left(z-z_{0}\right)^{1 / r}\right)^{-1} g .
$$

If $\operatorname{det} D^{\mathrm{I}}\left(z_{0}\right)=0$, we can choose $g$ with $|g|=1$ in such a way that $\lim v_{1}^{\mathrm{I}}=\infty$ for $z \rightarrow z_{0}$. This is not possible if the approximation is stable because (8.4) implies $\left|v_{1}^{\mathrm{T}}\right| \leqq$ const $|g|$.

We shall now consider Definition 3.2. For that purpose, we shall discuss a special class $R$ of approximations which is defined by

Definition 10.1. $R$ is the class of approximations for which the normal form of $M$ does not contain any block of type (9.4).

There is no difficulty to characterize this class algebraically.

THEOREM 10.1. Let $z_{i}$ denote the eigenvalues of (5.3). The approximation belongs to $R$ if and only if $\left|z_{j}\right|=1$ for some $\xi=\xi_{0}$ implies $\partial z_{j}\left(\xi_{0}\right) / \partial \xi \neq 0$.

Proof. If $\partial z_{j}\left(\xi_{0}\right) / \partial \xi \neq 0$, then we can consider $\kappa=e^{i \xi}$ in a neighbourhood of $\xi_{0}$ as a function of $z$ and get for the corresponding eigenvalue $\kappa=\kappa(z)$ of $M$ an expansion

$$
\kappa(z)=e^{i \xi_{0}}+\left(z-z_{0}\right)\left(\partial z\left(\xi_{0}\right) / \partial e^{i \xi}\right)^{-1}+\cdots .
$$

This is impossible if $\kappa(z)$ is an eigenvalue of a block $M_{i}$ with $M_{i}^{(0)}$ of type (9.4).

THEOREM 10.2. If the approximation is dissipative, then it belongs to $R$.

Proof. We need only to consider a neighbourhood of $\xi=0$, because, by assumption, $\left|z_{j}(\xi)\right|<1$ for $0<|\xi| \leqq \pi$. Consistency implies that there are precisely $n$ eigenvalues $z_{i}$ of (5.3) with $z_{i}(0)=1$ and for these eigenvalues we have

$$
z_{i}=1+i(k / h) a_{i} \xi+O\left(\xi^{2}\right) .
$$

The other eigenvalues are smaller than one in absolute value. This proves the theorem.

Let $z=z_{0},\left|z_{0}\right|=1$, be fixed and assume that the approximation belongs to $R$. Introducing again $y=T w$ as a new variable, we can write the Eq. (8.1) in the form

$$
y_{\nu+1}=\left(\begin{array}{c}
y_{\nu+1}^{(1)} \\
\vdots \\
y_{\nu+1}^{(4)}
\end{array}\right)=\left(\begin{array}{cccc}
L_{1} & 0 & 0 & 0 \\
0 & L_{2} & 0 & 0 \\
0 & 0 & N_{1} & 0 \\
0 & 0 & 0 & N_{2}
\end{array}\right)\left[\begin{array}{c}
(1) \\
\vdots \\
y_{\nu}^{(4)}
\end{array}\right]+T G,
$$

where $L_{1}, N_{1}$ have the properties defined in (9.3), and $L_{2}, N_{2}$ consist of blocks as described in Theorem 9.3.

The boundary conditions can again be written in the form (10.2) with $y^{\mathrm{I}}=$ $\left(y^{(1)}, y^{(2)}\right)^{\prime}, y^{\mathrm{II}}=\left(y^{(3)}, y^{(4)}\right)^{\prime}$ and we may, without restriction, assume that they have the form

$$
\left(\begin{array}{cc}
D_{11} & 0 \\
0 & D_{22}
\end{array}\right)\left(\begin{array}{l}
y_{1}^{(1)} \\
y_{1}^{(2)}
\end{array}\right)+\left(\begin{array}{ll}
E_{11} & E_{12} \\
E_{21} & E_{22}
\end{array}\right)\left(\begin{array}{l}
y_{1}^{(3)} \\
y_{1}^{(4)}
\end{array}\right)=g+R(G) .
$$

THEOREM 10.3. If an approximation of class $R$ is stable according to Definition 3.2, then there is a constant $K_{4}$ such that 


$$
\begin{aligned}
\left|D_{22}^{-1}\right| & \leqq K_{4}, \\
\left|D_{11}^{-1} E_{11}\right| & \leqq K_{4}\left|z-z_{0}\right|^{-1}, \\
\left|D_{11}^{-1} E_{12}\right| & \leqq K_{4} .
\end{aligned}
$$

Proof. Let $G \equiv 0$. Then $y_{1}^{(3)}=y_{1}^{(4)}=0$ and, therefore, $y_{\nu}^{(2)}=L_{2}^{\nu-1} D_{22}^{-1} g^{(2)}$. If $(10.12)$ does not hold, then $D_{22}^{-1}$ grows at least like $\left(z-z_{0}\right)^{-1}$ and the inequality (8.5) cannot be fulfilled. In the same way, we can prove that $\left|D_{11}^{-1}\right| \leqq$ const $\left|z-z_{0}\right|^{-1}$.

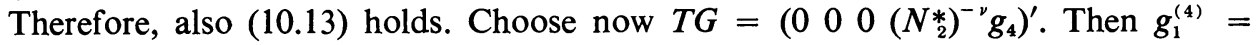
$\left(N_{2} N_{2}^{*}-I\right)^{-1} g_{4} \sim(|z|-1)^{-1} g_{4}$ and (8.5) cannot be fulfilled if (10.14) does not hold.

11. The Generalized Energy Method. We consider now the Eqs. (5.1) and (5.2) and write them again in the form (8.1), (8.3):

$$
w_{\nu+1}=M w_{\nu}+G_{\nu}, \quad S w_{1}=g+R(G) .
$$

THEOREM 11.1. Assume that there is a matrix $H(z)$ which for all $z$ with $|z|>1$ has the following properties:

(1) $H(z)$ is Hermitian and uniformly bounded.

(2) There is a constant $\delta_{1}>0$, such that, for all $z$ with $|z|>1$,

$$
|z|\left(M^{*} H M-H\right) \geqq \delta_{1}(|z|-1) .
$$

(3) There are constants $\delta_{j}>0, j=2,3$, such that, for all $w_{1}$ which fulfill the boundary conditions $S w_{1}=g+R(G)$,

$$
w_{1}^{*} H w_{1} \geqq \delta_{2}\left|w_{1}\right|^{2}-\delta_{3}\left(|g|^{2}+|R(G)|^{2}\right) .
$$

Then, the approximation is stable in the sense of Definition 3.3.

Proof. Let $N>0$ be any natural number. Then, we get, from (11.1),

$$
\begin{array}{r}
\operatorname{Re}\left(\sum_{\nu=1}^{N-1} w_{\nu+1}^{*} H M w_{\nu} h\right)-\sum_{\nu=1}^{N-1} w_{\nu+1}^{*} H w_{\nu+1} h=-\operatorname{Re}\left(\sum_{\nu=1}^{N-1} w_{\nu+1}^{*} H G_{\nu} h\right), \\
\sum_{\nu=1}^{N-1} w_{\nu}^{*} M^{*} H M w_{\nu} h-\operatorname{Re}\left(\sum_{\nu=1}^{N-1} w_{\nu}^{*} M^{*} H w_{\nu+1} h\right)=-\operatorname{Re}\left(\sum_{\nu=1}^{N-1} w_{\nu}^{*} M H G h\right) .
\end{array}
$$

Adding the last two equations gives us

$$
\begin{aligned}
\sum_{\nu=1}^{N-1} w_{\nu}^{*}\left(M^{*} H M-H\right) w_{\nu} h & +h w_{1}^{*} H w_{1}-h w_{N}^{*} H w_{N} \\
& =-\operatorname{Re}\left(\sum_{\nu=1}^{N-1}\left(w_{\nu+1}^{*} H G_{\nu}+w_{\nu}^{*} M H G_{\nu}\right) h\right) .
\end{aligned}
$$

Therefore, for $N \rightarrow \infty$, we get, from (11.2) and (11.3),

$$
\begin{aligned}
& \delta_{1}(|z|-1)\|w\|_{h}^{2}+h \delta_{2}|z|\left|w_{1}\right|^{2} \\
& \leqq h \delta_{3}|z||g|^{2}+h \delta_{3}|z||R(G)|^{2}+|z| \text { const }\|G \mid\|_{h} \cdot\|w\|_{h},
\end{aligned}
$$

i.e.,

$$
\begin{aligned}
\frac{1}{2} \delta_{1}(|z| & -1)\|w\|_{h}^{2}+h \delta_{2}|z|\left|w_{1}\right|^{2} \\
& \leqq h \delta_{3}|z||g|^{2}+h \delta_{3}|z||R(G)|^{2}+\text { const } \delta_{1}^{-1} \cdot \frac{|z|^{2}}{2}(|z|-1)^{-1}\|G\|_{h}^{2}
\end{aligned}
$$

and the inequality (8.4) follows without difficulties. 
There is no difficulty in constructing $H$ for $|z|>1+\eta$.

THEOREM 11.2. Let $\eta>0$ be a constant. If the Ryabenkii-Godunov condition is fulfilled, then we can construct $H$ for $1+\eta \leqq|z| \leqq\left|z_{\infty}\right|$.

Proof. It follows from Theorem 9.1 that there is a transformation $T(z)$, such that

$$
T(z) M(z) T(z)^{-1}=\left(\begin{array}{ll}
L & 0 \\
0 & N
\end{array}\right), \quad L^{*} L \leqq(1-\delta) I, N^{*} N>(1+\delta) I,
$$

for $1+\eta \leqq|z| \leqq\left|z_{\infty}\right|$.

We choose now

$$
H=T^{*}\left(\begin{array}{cc}
-c I & 0 \\
0 & I
\end{array}\right) T
$$

where $c$ with $0<c \leqq 1$ is a constant to be chosen later. Then

$$
|z|\left(M^{*} H M-H\right)=|z| T^{*}\left(\begin{array}{cc}
c\left(I-L^{*} L\right) & 0 \\
0 & N^{*} N-I
\end{array}\right) T \geqq|z| c \delta
$$

and, therefore, (11.2) is fulfilled. We also have

$$
w_{1}^{*} H w_{1}=y_{1}^{*}\left(\begin{array}{cc}
-c I & 0 \\
0 & I
\end{array}\right) y_{1}=-c\left|y_{1}^{\mathrm{I}}\right|^{2}+\left|y_{1}^{\mathrm{II}}\right|^{2}
$$

and, by (10.2) and Lemma 10.2, we can easily choose $c$ in such a way that (11.3) is fulfilled. This proves the theorem and, in addition, also Theorem 5.3.

We shall now prove Theorem 5.4. For that purpose, we assume that $h^{-1}=N$, $N$ natural number, and consider (8.1) for $\nu=1,2, \cdots, N$, with boundary conditions

$$
S(0) w_{1}=g(0)+R_{0}(G), \quad S(1) w_{N}=g(1)+R_{1}(G) .
$$

Let $\varphi \in C^{\infty}$ denote a monotone function with

$$
\begin{aligned}
\varphi=\varphi(x, \epsilon) & =0 & & \text { for } x \leqq \frac{1}{4} \epsilon, \\
& =1 & & \text { for } x \geqq \frac{1}{2} \epsilon
\end{aligned}
$$

and let $\psi=1-\varphi$. Then, we can write the Eqs. (8.1), (11.6) in the form

$$
\begin{aligned}
(\varphi w)_{v+1} & =M(\varphi w)_{\nu}+\left(\varphi_{\nu+1}-\varphi_{\nu}\right) w_{\nu+1}+\varphi_{\nu} G_{v}, \\
S(1)(\varphi w)_{N} & =g(1)+R_{1}(G), \\
(\psi w)_{\nu+1} & =M(\psi w)_{\nu}+\left(\psi_{\nu+1}-\psi_{\nu}\right) w_{\nu+1}+\psi_{\nu} G_{\nu}, \\
S(0)(\psi w)_{1} & =g(0)+R_{0}(G) .
\end{aligned}
$$

If we neglect the terms $\left(\varphi_{v+1}-\varphi_{v}\right) w_{\nu+1},\left(\psi_{\nu+1}-\psi_{\nu}\right) w_{\nu+1}$, then we can consider (11.7) and (11.8) as the resolvent equation for the left and right quarter-plane problem, respectively. These are, by assumption, stable and, therefore, the estimate (8.4) or (8.5) holds for the system

$$
\left(\begin{array}{ll}
\varphi & w \\
\psi & w
\end{array}\right)_{\nu+1}=M\left(\begin{array}{ll}
\varphi & w \\
\psi & w
\end{array}\right)_{\nu}+\left(\begin{array}{ll}
\varphi & w \\
\psi & w
\end{array}\right)_{\nu} .
$$


Now, the terms $\left(\varphi_{\nu+1}-\varphi_{\nu}\right) w_{\nu},\left(\psi_{\nu+1}-\psi_{\nu}\right) w_{\nu}$ can be considered as perturbations of order $k$ and, therefore, Theorem 5.4 follows from Theorem 4.3.

12. Dissipative Approximations. We assume that the approximation belongs to the class $R$ and that the Ryabenkii-Godunov condition is fulfilled. By Theorem 11.2, we need to construct $H$ only for $1 \leqq|z| \leqq 1+\eta$. Theorem 10.2 implies that there is a transformation $T=T(z)$ such that

$$
T M T^{-1}=\left(\begin{array}{cccc}
L_{1} & 0 & 0 & 0 \\
0 & L_{2} & 0 & 0 \\
0 & 0 & N_{1} & 0 \\
0 & 0 & 0 & N_{2}
\end{array}\right) .
$$

Here $L_{1}, N_{1}$ are of type (9.3) and $L_{2}, N_{2}$ consist of blocks as described in Theorem 9.3. Let $y_{\nu}=T w_{v}$. Then, we can write the boundary conditions $S w_{1}=g+R(G)$ in the form (10.11). If for all $|z|$ with $1 \leqq|z| \leqq 1+\eta$ the determinants $\operatorname{det}\left|D_{i i}\right| \neq 0, i=1,2$, then there exists a constant $K$ such that

$$
\left|y_{1}^{(1)}\right|+\left|y_{1}^{(2)}\right| \leqq K\left(\left|y_{1}^{(3)}\right|+\left|y_{1}^{(4)}\right|+|g|+R(G)\right) .
$$

Choose now

$$
H=T^{*}\left(\begin{array}{cccc}
-c I & 0 & 0 & 0 \\
0 & -c I & 0 & 0 \\
0 & 0 & I & 0 \\
0 & 0 & 0 & I
\end{array}\right) T .
$$

Then there is no difficulty in showing that the inequalities (11.2) and (11.3) are fulfilled if we choose the constant $c>0$ sufficiently small. Therefore, the approximation is stable according to Definition 3.3. This proves Theorem 5.1 for dissipative approximations.

If there are some $z_{0 j}$ with $\left|z_{0 j}\right|=1$ for which only the inequalities $(10.12)-(10.14)$ hold, then we have, instead of (12.1),

$$
\begin{aligned}
& \left|y_{1}^{(2)}\right| \leqq K\left(\left|y_{1}^{(3)}\right|+\left|y_{1}^{(4)}\right|+|g|+|R(G)|\right), \\
& \left|y_{1}^{(1)}\right| \leqq K \sum_{j}\left|z-z_{0 j}\right|^{-1}\left(\left|y_{1}^{(3)}\right|+|g|+R(G)\right)+K\left|y_{1}^{(4)}\right| .
\end{aligned}
$$

In this case, we choose

$$
H=T^{*}\left(\begin{array}{cccc}
-c\left(\frac{|z|-1}{|z|}\right)^{2} I & 0 & 0 & 0 \\
0 & -c\left(\frac{|z|-1}{|z|}\right) I & 0 & 0 \\
0 & 0 & I & 0 \\
0 & 0 & 0 & \left(\frac{|z|-1}{|z|}\right) I
\end{array}\right) T
$$


and, by introducing $y_{\nu}=T w_{\nu}$ into (11.4), a simple calculation shows that we can fulfill the inequality $(8.5)$ for sufficiently small $c$. Therefore, the approximation is stable in the sense of Definition 3.2. This proves Theorem 5.2.

13. Nondissipative Approximations. In this section, we want to prove Theorem 5.1 for nondissipative approximations. Let $z_{0}$ with $\left|z_{0}\right|=1$ be fixed. By Theorems 9.1-9.3, there is, in a neighbourhood of $z_{0}$, an analytic transformation $T=T(z)$ such that

$$
T M T^{-1}=\left(\begin{array}{ccccc}
M_{1} & 0 & & \cdots & 0 \\
0 & M_{2} & 0 & \cdots & 0 \\
\vdots & & & & \vdots \\
0 & \cdots & 0 & & M_{l}
\end{array}\right),
$$

where the matrices $M_{j}$ have the same properties as described in Theorems 9.1-9.3. We now choose $H$ in the form

$$
H=T^{*}\left(\begin{array}{ccccc}
R_{1} & 0 & & \cdots & 0 \\
0 & R_{2} & 0 & \cdots & 0 \\
\vdots & & & & \vdots \\
0 & \cdots & 0 & & R_{l}
\end{array}\right) T
$$

and get, from Lemma 10.3,

LEMMA 13.1. Let the determinant condition (10.3) be fulfilled for $|z| \geqq 1$. An $H$ which fulfills the conditions of Theorem 11.1 and thus proves stability in the sense of Definition 3.3 can be constructed in a neighbourhood of $z=z_{0}$ if, for every constant $c>0$, there is a constant $\delta_{1}>0$ and a Hermitian $R_{i}(z)$ such that

$$
\begin{gathered}
|z|\left(M_{i}^{*} R_{j} M_{j}-R_{i}\right) \geqq \delta_{1}(|z|-1), \\
\left(y_{1}^{(j)}\right)^{*} R_{i} y_{1}^{(j)} \geqq-c\left|y_{1}^{(j 1)}\right|^{2}+\left|y^{(j 2)}\right|^{2} .
\end{gathered}
$$

Here $y^{(i)}=\left(y^{(i 1)}, y^{(j 2)}\right)$ is defined by (10.5) and (10.8).

We shall now show how $R_{i}$ satisfying these conditions can be constructed. In the same way as earlier, we can prove that for $j=1$, and $j=l^{*}+1, \cdots, l$,

$$
R_{i}=\left(\begin{array}{cc}
-c I & 0 \\
0 & I
\end{array}\right)
$$

fulfills the conditions (13.1), (13.2).

For $j=2, \cdots, l^{*}$, we can write the corresponding $M_{j}$ as exponential functions

$$
M_{j}(z)=e^{\tilde{M}_{i}(z)}
$$

with

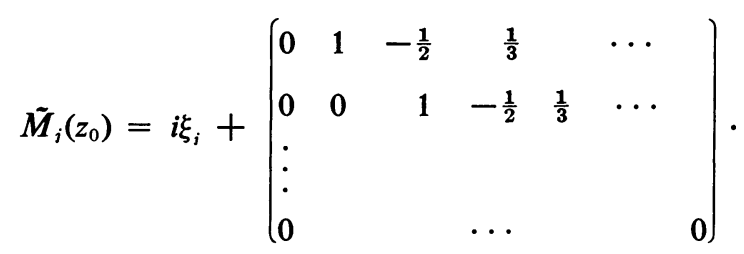


Furthermore, if $\tilde{\kappa}_{j \tau}, \kappa_{j \tau}$ denote the eigenvalues of $\tilde{M}_{j}, M_{i}$, respectively, then $e^{\tilde{x}_{i r}}=\kappa_{j \tau}$. From Lemma 9.1 and Cramer's rule, it follows that there are constants $c_{i}>0$ such that

$$
\begin{aligned}
K(|z|-1)^{-1} & \geqq \sup _{\varphi}\left|\left(M_{j}-e^{i \varphi} I\right)^{-1}\right| \geqq c_{1} \sup _{\varphi}\left|\prod_{\tau}\left(\kappa_{j \tau}-e^{i \varphi}\right)^{-1}\right| \\
& =c_{1} \sup _{\varphi}\left|\prod_{\tau}\left(e^{\tilde{x}_{j \tau}}-e^{i \varphi}\right)^{-1}\right| \geqq c_{2} \sup _{\varphi}\left|\prod_{\tau}\left(\tilde{\kappa}_{i \tau}-i \varphi\right)^{-1}\right| \\
& \geqq c_{3} \sup _{\varphi}\left|\left(\tilde{M}_{i}-i \varphi I\right)^{-1}\right| .
\end{aligned}
$$

We have thus proved that, in a neighbourhood of $z_{0}$,

$$
\sup _{\varphi}\left|\left(\tilde{M}_{i}-i \varphi I\right)^{-1}\right| \leqq \text { const }|| z|-1|^{-1}, \quad|z|>1 .
$$

By assumption, the approximation is nondissipative. Therefore it is stable for the Cauchy problem, not only for $t \rightarrow+\infty$ but also for $t \rightarrow-\infty$. Thus, the resolvent condition (9.12) and (13.5) hold also for $|z|<1$. Now let $z=e^{s}$ and $z_{0}=e^{s o}$. Then we have proved

LEMMA 13.2. The matrices $M_{i}$ are in a neighbourhood of $s_{0}$ analytic functions of $s_{0}$ and the double-sided resolvent condition

$$
\sup _{\varphi}\left|\left(\tilde{M}_{i}-i \varphi I\right)^{-1}\right| \leqq \text { const }|\operatorname{Re} s|^{-1}
$$

holds.

In a similar way as in [4], Ralston [6] has shown that (13.4) and (13.6) imply that for every constant $c>0$ there is a constant $\delta_{1}>0$ and Hermitian $\tilde{R}_{i}(z)$ such that

$$
\begin{aligned}
\tilde{R}_{j} \tilde{M}_{j}+\tilde{M}_{i}^{*} \tilde{R}_{i} & \geqq \delta_{1}(|z|-1) \approx \delta_{1} \operatorname{Re} s, \quad z=e^{s}, \\
\left(y_{1}^{(j)}\right)^{*} R_{j} y_{1}^{(j)} & \geqq-c\left|y_{1}^{(j 1)}\right|^{2}+\left|y_{1}^{(j 2)}\right|^{2} .
\end{aligned}
$$

We shall now show that we can choose $R_{i}=\tilde{R}_{i}$. Consider the differential equation

$$
d y / d x=\tilde{M}_{j} y, \quad 0 \leqq x \leqq 1,
$$

then

$$
\begin{aligned}
y^{*}(0)\left(e^{\tilde{M}_{j}^{*}} \tilde{R}_{j} e^{\tilde{M}_{j}}-\tilde{R}_{j}\right) y(0) & =2 \operatorname{Re}\left(\int_{0}^{1} y^{*} \tilde{R}_{j} d y / d x d x\right) \\
& =2 \operatorname{Re}\left(\int_{0}^{1} y^{*} \tilde{R}_{i} \tilde{M}_{j} y d x\right) \geqq 2 \delta_{1}(|z|-1)|y(0)|^{2} .
\end{aligned}
$$

Observing that $y(0)$ is arbitrary, we see that (13.1) is fulfilled. The inequality (13.2) is also fulfilled by (13.8), and we have thus been able to construct $R_{j}$ for $j=1, \cdots, l$, satisfying the conditions of Lemma 13.1. This proves Theorem 5.1 also for the nondissipative case.

Department of Computer Sciences

University of Uppsala

Uppsala, Sweden

1. H.-O. KREISS, "Stability theory for difference approximations of mixed initial boundary value problems. I," Math. Comp., v. 22, 1968, pp. 703-714. MR 39 \#2355. 
2. H.-O. KREISS, "Difference approximations for mixed initial boundary value problems," Proc. Roy. Soc. London Ser. A, v. 323, 1971, pp. 255-261.

3. H.-O. KREISS, "Difference approximations for the initial-boundary value problem for hyperbolic differential equations," Numerical Solutions of Nonlinear Differential Equations (Proc. Adv. Sympos., Madison, Wis., 1966), Wiley, New York, 1966, pp. 141-166. MR 35 \#5156.

4. H.-O. KreIss, "Initial boundary value problems for hyperbolic systems," Comm. Pure Appl. Math., v. 23, 1970, pp. 277-298.

5. S. OsHeR, "Stability of parabolic difference approximations to certain mixed initial boundary value problems," Math. Comp., v. 26, 1972, pp. 13-39.

6. J. V. Ralston, "Note on a paper of Kreiss," Comm. Pure Appl. Math., v. 24, 1971, pp. 759-762. 